\title{
Three-dimensional MHD simulation of the 2008 December 12 coronal mass ejection: from the Sun to Interplanetary space
}

\author{
Man Zhang ${ }^{1, *}$, Xue Shang Feng ${ }^{1,2}$, and Li Ping Yang ${ }^{1}$ \\ ${ }^{1}$ SIGMA Weather Group, State Key Laboratory for Space Weather, National Space Science Center, \\ Chinese Academy of Sciences, 100190 Beijing, PR China \\ ${ }^{2}$ HIT Institute of Space Science and Applied Technology, 518055 Shenzhen, PR China
}

Received 24 October 2018 / Accepted 4 August 2019

\begin{abstract}
A three-dimensional time-dependent, numerical magnetohydrodynamic simulation is performed to investigate the propagation of a coronal mass ejection that occurred on 12 December 2008. The background solar wind is obtained by using a splitting finite-volume scheme based on a six-component grid system in spherical coordinate, with Parker's one-dimensional solar wind solution and measured photospheric magnetic fields as the initial values. A spherical plasmoid is superposed on the realistic ambient solar wind to study the 12 December 2008 coronal mass ejection event. The plasmoid is assumed to have a spheromak magnetic structure with a high-density, high-velocity, and high-pressure near the Sun. The dynamical interaction between the coronal mass ejection and the background solar wind flow is then investigated. We compared the model results with observations, and the model provide a relatively satisfactory comparison with the Wind spacecraft observations at $1 \mathrm{AU}$. We also investigated the numerical results assuming different parameters of the CME, we find that initial magnetic fields in the CME have a larger influence on the solar wind parameters at the Earth.
\end{abstract}

Keywords: magnetohydrodynamics / coronal mass ejection / flux rope

\section{Introduction}

Coronal mass ejections (CMEs) have been defined as largescale expulsions of magnetized plasma from the corona seen as bright arcs in coronagraphs. During a typical CME, $10^{15}-10^{16} \mathrm{~g}$ of plasma is hurled into interplanetary space with a kinetic energy the order of $10^{31}-10^{32}$ ergs. It is well known that CMEs are the most important drivers of various types of space weather disturbance, especially when they contain a prolonged southward directed magnetic fields. Since the southward interplanetary magnetic field is the primary cause of geoeffective storms, the problem of CME propagation from the corona to $1 \mathrm{AU}$ is a important component of space weather. The properties of a CME at $1 \mathrm{AU}$ will be determined by how it interacts with its ambient solar wind during propagation in interplanetary space (Iv et al., 2004a, 2004b; Lugaz et al., 2011; Zhou \& Feng, 2013; Shen et al., 2014; Shiota \& Kataoka, 2016).

In order to realistically capture the properties of CMEs, the numerical simulations have become one of the primary tools to investigate the propagation of CMEs and their interaction with

\footnotetext{
*Corresponding author: mzhang@spaceweather.ac. cn
}

the interplanetary medium. Solutions to the magnetohydrodyamics (MHD) equations are the only self-consistent mathematical description of the Sun-Earth space environment that can span the necessary range of length-scales to provide a truly global model of the system (Iv et al., 2004a). Placing the inner boundary of the simulation domain beyond $18 R_{s}$ has considerable advantages from a numerical point of view, since all characteristic waves are propagating in the domain and the variables can be specified at the boundary (Jacobs \& Poedts, 2011). Han et al. (1988) have studied a MHD disturbance propagation from $18 R_{s}$ to the Earth using a threedimensional (3D) MHD model, and it is the first application to the field of interplanetary physics. Odstrćil \& Pizzo (1999a, 1999b) studied the evolution of CMEs launched at several heliographic positions into a tilted-dipole ambient solar wind beyond $21.5 R_{s}$ using 3D MHD numerical model. Vandas et al. (2002) modelled an interplanetary flux rope from $30 R_{s}$ to $350 R_{s}$, its evolution and propagation through interplanetary space are studied using 3D MHD self-consistent numerical simulations. Odstrćil et al. (2005) simulated the propagation of the 12 May 1997 interplanetary CME from 0.1 AU to $1.7 \mathrm{AU}$, where the transient disturbance was determined from the cone model. They analyzed possible interactions of the 
CME propagating in various steady state and evolving configurations of the background solar wind using this model. Kataoka et al. (2009) used a 3D MHD to reconstruct a interplanetary CME that occurred on 13 December 2006 from $50 R_{s}$ to 1 AU. The simple CME model is assumed to have a spheromak magnetic structure with a high-density, high-velocity, and hightemperature at the inner boundary. Shiota \& Kataoka (2016) report a newly developed MHD simulation of interplanetary propagation of a series of multiple CMEs with internal spheromak-type magnetic fields. All of these studies, however, were limited by having a lower boundary beyond $18 R_{s}$. Since there are little or no observable parameters at these large coronal distances to constrain the inner boundary conditions of the interplanetary CME, it seems necessary to use models extent from Sun to Earth to study the propagation of CMEs.

Iv et al. (2004a, 2006) presented a 3D MHD model describing the time-dependent expulsion and propagation of a $\mathrm{CME}$ from the solar corona to $1 \mathrm{AU}$, and the $\mathrm{CME}$ is driven by superimposing a Gibson-Low magnetic flux rope inside the coronal streamer belt. Chané et al. (2006) have simulated some CMEs from the Sun up to $1 \mathrm{AU}$ and investigated the effect of the initial magnetic polarity on the evolution and on the geo-effectiveness of CMEs. The CMEs are simulated by means of a very simple model: a high density and high pressure magnetized plasma blob is superposed on a background steady state solar wind. Jacobs et al. (2007) showed a comparison between 2.5D and 3D simulations of CMEs, the CMEs are triggered by a simple "density-driven" model. Lugaz et al. (2007) showed a 3D compressible MHD simulation of the three interacting CMEs from Sun to Earth, and the ejections are initiated using out-of-equilibrium semicylindrical flux ropes with a size smaller than the active region. Wu et al. (2007a, 2007b) used Hakamada-Akasofu-Fry code version 2 (HAFv.2) + 3DMHD to study the global interplanetary CME propagation in a realistic 3D solar wind structure from Sun to Earth. They use solar magnetogram data to simulate the solar wind structure from the photosphere to $2.5 R_{s}$, and the HAFv. 2 code was then used from $2.5 R_{s}$ to provide the inner boundary at $18 R_{s}$ for the 3D MHD code to calculate solar wind to $1 \mathrm{AU}$ and beyond. Zuccarello et al. (2012a) performed a 2.5D numerical MHD simulations to investigated the deflections of CMEs, and the CME is initiated by applying localized photospheric shearing motions. Zuccarello et al. (2012b) presented a numerical MHD data-inspired model suitable for the simulation of the CME initiation and their early evolution. In their simulation, a force-free magnetic field configuration is obtained as a consequence of the applied twisting motions, which has the same chirality as the investigated active region. Zhou et al. (2012) and Zhou \& Feng (2013) used a spherical plasmoid to interpret the propagation and deflection of CMEs from the corona to interplanetary space by a 3D numerical MHD simulation. Shen et al. (2014) presented a data-constrained 3D MHD simulation of the evolution of the CME in a realistic ambient solar wind from Sun to Earth by using the 3D corona interplanetary total variation diminishing (COIN-TVD) MHD code.

Numerical simulations for CMEs are necessary and complementary to the observations. In this article, we use a 3D MHD to study the propagation of a CME that occurred on 12 December 2008 from Sun to Earth. By splitting the MHD equations into a fluid part and a magnetic part, a finite volume method is used for the fluid part and the constrained-transport method
(CT) is used for the magnetic induction part for the magnetic divergence cleaning (Ziegler, 2004, 2011). For details of the scheme, please refer to Feng et al. (2014). Once the steady state solar wind is produced, a very simple $3 \mathrm{D}$ spherical plasmoid model is superposed on the background solar wind to initiate the CME, which has a high speed, density, and pressure compared to the solar wind ambient. The CME contains a spheromak-type magnetic field which is a linear force-free field in a completely isolated sphere (Kataoka et al., 2009). The exact characteristics and morphology of the magnetic field inside CMEs are under debate, but Gibson \& Fan (2008) believed that the spheromak-type magnetic field may be a natural product of a plasmoid ejected from a solar flare via reconnection. Then, the $3 \mathrm{D}$ propagation and dynamical interaction of the $\mathrm{CME}$ with the background solar wind flow between solar surface and $1 \mathrm{AU}$ is investigated. In order to produce a relatively satisfactory comparison with the Wind spacecraft, we adjusted the CME initial parameters (density, magnetic field, velocity, and pressure) in the numerical simulation to yield a best fit with observations. We also investigated the numerical results assuming different parameters of the CME, we find that initial magnetic fields in the CME have a larger influence on the solar wind parameters at the Earth.

The organization of the paper is as follows. We give a brief description of observational properties of the 12 December 2008 CME event in Section 2. The conservative form of the MHD equations and the scheme is described in Section 3. Section 4 is devoted to the spherical plasmoid CME model. Section 5 introduces the simulation results of the background solar wind and the CME event. Finally, some conclusions are given in Section 6.

\section{Observation of the 12 December 2008 CME event}

The 12 December 2008 CME is generally regarded as the first geoeffective CME observed during the STEREO mission, and it has been studied by many authors (Davis et al., 2009; Byrne et al., 2010; Lugaz, 2010; Ying et al., 2010; Deforest et al., 2011, 2012; Howard \& Deforest, 2012; Deforest et al., 2013). An erupting prominence in the northern hemisphere was observed by STEREO on 12 December 2008, which is visible from 03:00 UT in SECCHI/Extreme Ultraviolet Imager (EUVI) images. This CME is induced by the prominence eruption (Byrne et al., 2010; Ying et al., 2010). The CME was observed first in the EUVI and COR1 fields of view at 2008 December 12 07:00 UT; the full initially-circular flux rope was visible to COR1 and COR2 from 2008 December 12 12:00C18:00 UT; and was fully visible in HI-1 and HI-2 from 2008 December 13 03:00 UT to the exit of the trailing edge from the HI-2 field of view early on 2008 December 18 (Deforest et al., 2013). At 2008 December 16, a structure of increased density and speed arrived at $1 \mathrm{AU}$, and a magnetic cloud was observed during the UT morning of 2008 December 17 by ACE (Davis et al., 2009) and Wind (Ying et al., 2010).

The CME was slow (transit time from $0 \mathrm{AU}$ to $1 \mathrm{AU}$ of four days) and produced no apparent forward shock. The projected speed according to the Coordinated Data Analysis Workshop 
LASCO CME catalog is $203 \mathrm{~km} / \mathrm{s}$. The central axis of the CME model pointed to $36^{\circ} \mathrm{N}, 10^{\circ} \mathrm{W}$, which is provided in the online catalog GMU CME/ICME List compiled by Phillip Hess and Jie Zhang at http://solar.gmu.edu/heliophysics/index.php/ GMU_CME/ICME_List (Hess \& Jie, 2017). These lists used data from the Wind and ACE satellites and/or the OMNI near-Earth database,taking into account all the recorded ICME data from these data bases.

\section{Model description}

In order to study the propagation of the 12 December 2008 CME Event from Sun to Earth, a representative MHD model of the steady state background solar wind is required. The MHD equations are splitted into the fluid part and the magnetic part, Harten-Lax-van Leer (HLL) scheme within a finite volume (FV) framework without Riemann solver or any kind of characteristic decomposition are used in this paper. To maintain the $\nabla \cdot \mathbf{B}$ constraint, the CT technique is used for magnetic field by utilizing a special discretization on a staggered grid (Ziegler, 2004, 2011; Feng et al., 2014). The ratio of specific heats $\gamma$ is varied from 1.05 to 1.5 along the heliocentric distance $r$, that is, $\gamma=1.05$ for $r / R_{s} \leq 5, \gamma=1.05+0.03\left(r / R_{s}-5\right)$ for $5<r / R_{s} \leq 20, \gamma=1.5$ for $r / R_{s}>20$. The details of computing the background solar wind were given by Feng et al. (2014).

The computational domain here covers $1 R_{s} \leq r \leq 230 R_{s}$, $0^{\circ} \leq \theta \leq 180^{\circ}$ and $0^{\circ} \leq \phi \leq 360^{\circ}$, where $r$ is the radial distance from solar center in units of $R_{s}$ is solar radius, $\theta$ is latitude angle and $\phi$ is longitude angle, respectively. Due to the radial expansion of this vast spherical shell domain, the MHD governing equations is discretized on highly non-uniform grids in order to resolve steep gradients in the solution. In order to mitigate this discrete or geometrical stiffness caused by disparate mesh cell widths, the following grid partitions are employed. For $1-75 R_{s}, N_{\theta}=120, N_{\phi}=240, \Delta r(i)=0.01 R_{s}$ if $r(i)<1.1 R_{s}$; $\Delta r(i)=\min \left(A \times \log _{10}(r(i-1)), \Delta \theta \times r(i-1)\right)$ with $A=$ $0.01 / \log _{10}$ (1.09) if $r(i)<3.5 R_{s} ; \Delta r(i)=\Delta \theta \times r(i-1)$ if $r(i)>3.5 R_{s}$. For 75-166 $R_{s}, N_{\theta}=190, N_{\phi}=380$ and $\Delta r(i)=\Delta \theta \times r(i-1)$. For 166-230 $R_{s}, N_{\theta}=260, N_{\phi}=520$ and $\Delta r(i)=\Delta \theta \times r(i-1) . N_{\theta}$ and $N_{\phi}$ represented the grids in latitude and longitude direction, respectively. The grid size is uniform in meridional and azimuth, with $\Delta \theta=\frac{\pi}{N_{\theta}}, \Delta \phi=\frac{2 \pi}{N_{\phi}}$. The radial grid size $\Delta r(i)$ are not uniform. The position of the Earth is $(x, y, z)=(-215,0,0) R_{s}$ at the beginning of the CME eruption, i.e., $r=215 R_{s}, \theta=90^{\circ}, \phi=180^{\circ}$. The parallel implementation in the whole computational domain is realized by domain decomposition of six-component grids based on the spherical surface and radial direction partition.

Initially, we specify the magnetic field by the line-of-sight photospheric magnetic data from the Wilcox Solar Observatory to produce a 3-D global magnetic field in the computational domain by the potential field source surface (PFSS) model. The initial distributions of plasma density $\rho$, pressure $p$, and the velocity $\boldsymbol{v}$ are given by Parker's solar wind flow (Parker, 1963). The temperature and number density on the solar surface are $T_{s}=1.3 \times 10^{6} \mathrm{~K}, \rho_{s}=1.5 \times 10^{8} \mathrm{~cm}^{-3}$. The inner boundary at $1 R_{s}$ is fixed for simplicity. Since the outer boundary in interplanetary is supersonic/super-Alfvénic region, the solar wind parameters at the outer boundary are set equal to the values at their nearest grid points in the computational domain. We then relax the system for 6.25 days until we obtain a steady state.

Here, we present the steady state solar wind numerical results from the 3D MHD numerical simulation, Figure 1 shows the MHD results for the magnetic fields and radial speed for the steady state solar wind solution, The left is on the meridional plane of $\phi=180^{\circ}-0^{\circ}$ from $1 R_{s}$ to $20 R_{s}$, and the right is on the equatorial plane from $20 R_{s}$ to $220 R_{s}$, the arrowheads denote the direction of the magnetic field and the color contours represent the radial solar wind speed. From this picture, it is seen that the magnetic field and radial speed possess a typical characteristic of solar minimum. The magnetic field lines extend to interplanetary space, and there appear fast solar wind flows at high latitudes and the slow solar wind are showed in the vicinity of the equator or heliospheric current sheet (HCS) region. The solar wind extends the interplanetary magnetic field outward into Archimedean spirals due to the solar rotation and the interplanetary magnetic field freezing-in effect.

\section{Spheromak CME model}

In this paper, a very simple spherical plasmoid model is superposed on the background solar wind to initiate the CME, which contains a high speed, high density and high pressure spherical plasmoid (Zhou et al., 2012; Zhou \& Feng, 2013). This kind of model do not have very complex features and gives us an easy way to study the evolution of the CME in the interplanetary space. The density, pressure and velocity profile of the initial perturbation are defined as follows:

$$
\begin{aligned}
& \rho=\rho_{0}+\rho_{\max }\left(1-\frac{a^{2}}{a_{\mathrm{mc}}^{2}}\right), \\
& v=v_{0}+v_{\max }\left(1-\frac{a^{2}}{a_{\mathrm{mc}}^{2}}\right), \\
& p=p_{0}+p_{\max }\left(1-\frac{a^{2}}{a_{\mathrm{mc}}^{2}}\right),
\end{aligned}
$$

where $a_{\mathrm{mc}}$ is the radius of the plasmoid, $a$ denotes the distance from the center of the plasmoid, and $\rho_{0}, v_{0}$ and $p_{0}$ are the density, velocity, and pressure of the background solar wind, respectively. $\rho_{\max }, v_{\max }$ and $p_{\max }$ are the maximum density, velocity and pressure added on top of the background solar wind, respectively.

A spheromak-type magnetic field which is a linear forcefree field in a completely isolated sphere is introduced as the simplest MC model in this paper (Kataoka et al., 2009). Wang et al. (2010a, 2010b) also described certain existence evidence on plasmoid in the magneto tail. In the present paper, the initial shape of the CME is assumed to be a sphere with the radius $a_{\mathrm{mc}}$ in local spherical coordinates $(\tilde{r}, \tilde{\theta}, \tilde{\phi})$ :

$$
\begin{gathered}
\tilde{B}_{\tilde{r}}(\tilde{r}, \tilde{\theta}, \tilde{\phi})=\left(2 B_{0} / \alpha \tilde{r}\right) j_{1}(\alpha \tilde{r}) \cos \tilde{\theta}, \\
\tilde{B}_{\tilde{\theta}}(\tilde{r}, \tilde{\theta}, \tilde{\phi})=-\left(B_{0} / \alpha \tilde{r}\right)\left[\sin (\alpha \tilde{r})-j_{1}(\alpha \tilde{r})\right] \sin \tilde{\theta}, \\
\tilde{B}_{\tilde{\phi}}(\tilde{r}, \tilde{\theta}, \tilde{\phi})=-B_{0} j_{1}(\alpha \tilde{r}) \sin \tilde{\theta},
\end{gathered}
$$



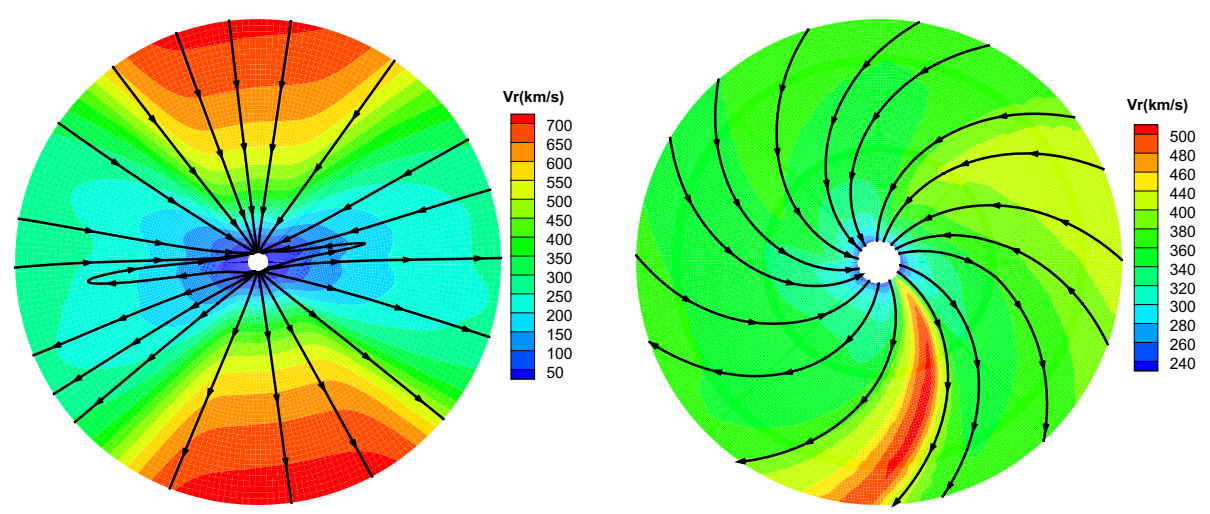

Fig. 1. Magnetic field and radial speed $v_{r}(\mathrm{~km} / \mathrm{s})$ for the steady state solar wind solution. The left is on the meridional plane of $\phi=180^{\circ}-0^{\circ}$ from $1 R_{s}$ to $20 R_{s}$, the right is on the equatorial plane from $20 R_{s}$ to $220 R_{s}$. The color contours represent the radial speed and streamlines denote the magnetic field lines.

where $B_{0}$ is a constant means the field strength of the spheromak. $j_{1}$ is the first-order spherical Bessel function:

$$
j_{1}(x)=\frac{\sin x-x \cos x}{x^{2}},
$$

$\alpha=4.493409458 a_{\mathrm{mc}}$ is the constant derived from the forcefree condition of $\nabla \times \boldsymbol{B}=\alpha \boldsymbol{B}$ with the boundary condition of $\tilde{B}_{\tilde{r}}=0$ at $\tilde{r}=a_{\mathrm{mc}}$, we only adopt the field within the isolated spherical shell $\tilde{r} \leq a_{\mathrm{mc}}$. At the spherical boundary $\tilde{r}=a_{\mathrm{mc}}$, the local radial $\widetilde{B}_{\tilde{r}}$ and azimuthal $\tilde{B}_{\tilde{\tilde{\sigma}}}$ components vanish and only the local zenithal component $\tilde{B}_{\tilde{\theta}}$ exists, while $\tilde{B}=0$ outside of the boundary. Therefore, the forces do not balance at the spherical boundary. The center of the CME is initially placed at $2 R_{s}$. The radius of the initial CME, $a_{\mathrm{mc}}$, is $0.8 R_{s}$. The observed CME is launched at N34W10 (i.e., $\theta=56^{\circ}, \phi=190^{\circ}$ ) to conform to the location of the CME.

The CME parameters are chosen as follows. The value of $v_{\max }$ is set to be $200 \mathrm{~km} / \mathrm{s}$. $\rho_{\max }$ and $p_{\max }$ are assumed to be ten and fifteen that of the ambient solar wind at the center of the CME, respectively. The maximal magnetic field strength $B_{0}$ is 5 Gauss. In order to find a better agreement with the in situ IMF observations, we rotate the polar axis of the CME toward the $y$ axis, the angle between the polar axis and the $y$ axis is defined as the inclination angle. The inclination angle is $150^{\circ}$ in our simulation. The initial plasma plasmoid has added to the corona $2.24 \times 10^{19} \mathrm{~J}$ of magnetic energy, $7.12 \times 10^{22} \mathrm{~J}$ of kinetic, and $4.19 \times 10^{23} \mathrm{~J}$ of thermal energy. The increase in total energy (magnetic + kinetic + thermal energy) is $4.26 \times 10^{23} \mathrm{~J}$. Here we estimate the energy injected into the computation domain by $\Delta E=E_{1}-E_{0}$, where $E_{1}\left(E_{0}\right)$ is the energy after (before) CME initiation. The magnetic, kinetic, and thermal energy values are calculated using the expressions: $E_{m}=\int \frac{B^{2}}{2} \mathrm{~d} V, E_{k}=\int \frac{\rho v^{2}}{2} \mathrm{~d} V, E_{t}=\int \frac{p}{\gamma-1} \mathrm{~d} V$. Figure 2 shows the 3D schematic picture of the initial magnetic field at $t=0$. Field lines are shown in color to illustrate the magnetic field strength. The color contours represent the radial magnetic field strength on the surface at $r=1 R_{s}$.

It should be noted that there would introduced some divergences at the interface of the spheromak CME model and the

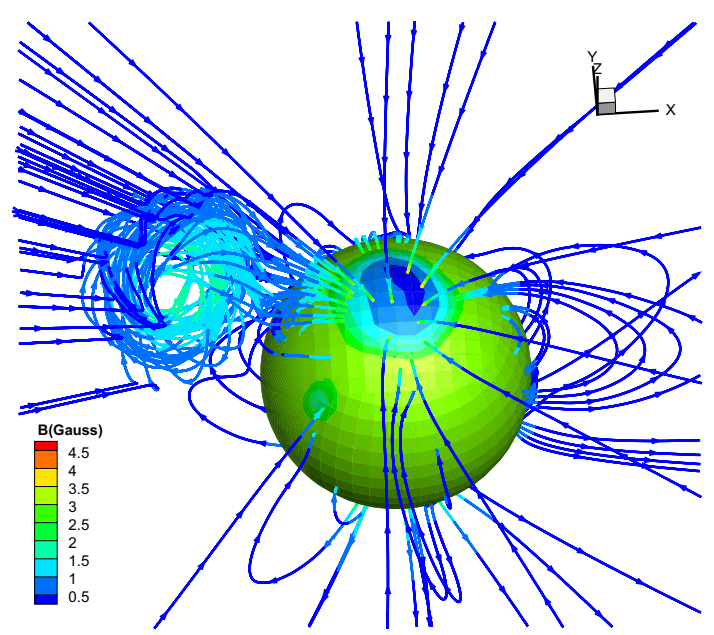

Fig. 2. Three dimensional view of the initial coronal magnetic field. Field lines are shown in color to illustrate the magnetic field strength. The color contours represent the radial magnetic field strength on solar surface.

magnetic field is non-solenoidal. The CT method can maintain constant the divergence of the magnetic field, but it can not smooth out or reduce the magnetic field divergence. Since the divergences generated as soon as the spheromak CME model supposed on the solar wind background. It is necessary to deal with the magnetic fields data derived from flux rope before they are supposed on the solar wind background. We now use the diffusion scheme to deal with the magnetic fields data to reduce the divergences. The diffusion scheme can be written as follows:

$$
\mathbf{B}^{k+1}-\mathbf{B}^{k}=\mu(\Delta x)^{2} \nabla\left(\nabla \cdot \mathbf{B}^{k}\right),
$$

where, $\mu=0.4,(\Delta x)^{2}=\frac{3}{\frac{1}{(\Delta r)^{2}}+\frac{1}{(r \Delta \theta)^{2}}+\frac{1}{(r \sin \theta \Delta \phi)^{2}}}$. Initially, $\mathbf{B}^{1}$ is the magnetic fields data derived from the spheromak CME model. If the $\max \left(\frac{\Delta x\left|\nabla \cdot \mathbf{B}^{k+1}\right|}{\left|\mathbf{B}^{k+1}\right|}\right)<10^{-3}$, the iteration is stopped. We then use $\mathbf{B}^{k+1}$ as the corrected magnetic fields data supposed on the solar wind background to simulate the CME. In our case, the number of iteration $k=80$. Figure 3 shows the the 

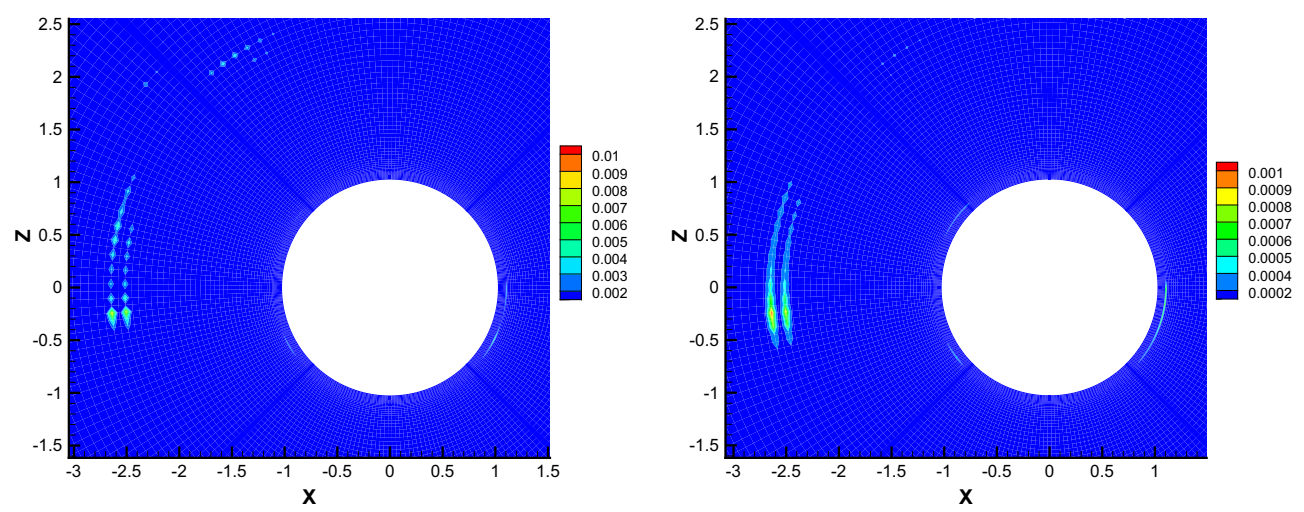

Fig. 3. The the error introduced by the non-solenoidal magnetic field on the Lorentz force (left) and magnetic field (right).

error introduced by the non-solenoidal magnetic field on the Lorentz force (left) and magnetic field (right). We defined $\frac{|(\nabla \cdot \mathbf{B}) \mathbf{B}|}{|(\nabla \times \mathbf{B}) \times \mathbf{B}|}$ as error introduced by the non-solenoidal magnetic field on the Lorentz force, and $\frac{\Delta x|\nabla \cdot \mathbf{B}|}{|\mathbf{B}|}$ as error introduced by the non-solenoidal magnetic field on the magnetic field. The error introduced by the non-solenoidal magnetic field on the Lorentz force and the magnetic field is about $10^{-3}, 10^{-4}$, respectively. So, it is the Lorentz force governing the CME's evolution and this remains a realistic representation for a magnetic flux rope, and the error introduced by the non-solenoidal magnetic field is acceptable in this paper.

In order to match the Wind data as the best fit as possible, some choices of the CME's parameters are empirical. We have tried 11 cases with different CME parameters to simulate the propagation of the CME, and we have chosen a best case which is shown above to reach a satisfactory comparison. We have also analysed how the CME's parameters can effected their output at $1 \mathrm{AU}$ in the next section.

\section{Results}

\subsection{Numerical results for 12 December 2008 CME}

This section present the results of the morphology and propagation of the CME, and the comparison between the numerical results and the in-situ data from Wind.

Figure 4 shows the $3 \mathrm{D}$ representation of the CME is shown at $3 \mathrm{~h}, 6 \mathrm{~h}, 20 \mathrm{~h}$ and $40 \mathrm{~h}$. The color code represents the velocity magnitude in the $x-y$ equatorial plane. The streamlines showed in black denote the magnetic field lines. The blue sphere in the first row is the Sun, and in the bottom row represent $20 R_{s}$. The top row shows the domain from $1 R_{s}$ to $20 R_{s}$, and the bottom show views from $20 R_{s}$ to $1 \mathrm{AU}$. From this figure, we can see that the magnetic field evolves nearly in self-similar manner. The plasmoid quickly expands because of the higher plasma density, speed, and magnetic pressure inside the CME. The radial and angular sizes of the CME increase with time. We can also find that nearly all of the magnetic field lines of spheromak CME are connected to the inner boundary from the beginning, that is because the magnetic field of the CME is introduced by a simple superposition on the background solar wind. In fact, the interplanetary magnetic fields connection between Sun and Earth is implied from strahl electrons observations (Feldman et al., 1978; Mccomas et al., 1989; Crooker et al., 2003; Gosling et al., 2004; Owens et al., 2008; Phillips et al., 2012; Kajdic et al., 2016).

Figure 5 shows the evolution of the spread angle of the $\mathrm{CME}$ in the ecliptic plane in time. We define the spread angle by means of the relative density plots on which we determine the contour where the relative density $\frac{\rho-\rho_{0}}{\rho_{0}}$ is $2 . \rho$ is the total density, and $\rho_{0}$ is the density of background solar wind. The spread angle of this contour measured from the solar center is defined as the spread angle of the CME. Though this definition is rather simple, at least it is based on mathematics and can reflect the angular size of the CME to some extent. The spread angle quickly evolves to $145^{\circ}$ just $1.5 \mathrm{~h}$ after the CME initiation. The quick increase of spread angle during the first $1.5 \mathrm{~h}$ of the CME's propagation is correlated with the rapid expansion due to the initial high speed, pressure, and plasma density in the plasmoid imposed on the solar wind ambient. The spread angle varies slightly from $145^{\circ}$ to $130^{\circ}$ between 1.5 and $4 \mathrm{~h}$, this may be due to the combined interaction of the CME with the background solar wind. After $4 \mathrm{~h}$, the spread angle remains almost around $130^{\circ}$, this means that the CME continues to expand during its propagating outward in interplanetary space.

Figure 6 shows time evolution of the CME in the $x-z$ meridional plane at $3 \mathrm{~h}$ and $6 \mathrm{~h}$. The streamlines showed in black denote the projection of the magnetic field lines. The color code in the top row are velocity and in the bottom row are relative density $\frac{\rho-\rho_{0}}{\rho_{0}}$. Figure 7 shows the evolution of the spatial difference in the characteristic speed $\lambda_{\text {max }}$ along the purple line in Figure 6. MHD flows contain four types of waves, the fast, slow, Alfvénic and entropy waves, which propagate at the characteristic speeds. The former two steepen and evolve into shock waves. Thus we measure steepening of them by $\lambda_{\max }$ to identify the shock fronts (Hanawa et al., 2008; Wada \& Liou, 2012). $\lambda_{\max }=\max \left(\Delta \lambda_{i}^{1}, \Delta \lambda_{i}^{2}, \Delta \lambda_{i}^{3}, \Delta \lambda_{i}^{4}, 0\right)$. where $\Delta \lambda_{i}^{1}=$ $\lambda_{i}^{1}-\lambda_{i+1}^{1}, \quad \Delta \lambda_{i}^{2}=\lambda_{i}^{2}-\lambda_{i+1}^{2}, \quad \Delta \lambda_{i}^{3}=\lambda_{i}^{3}-\lambda_{i+1}^{3}$, $\Delta \lambda_{i}^{4}=\lambda_{i}^{4}-\lambda_{i+1}^{4} . \quad \lambda_{i}^{1}=v_{r}+c_{f}, \quad \lambda_{i}^{2}=v_{r}-c_{f}, \quad \lambda_{i}^{3}=v_{r}+c_{s}$, $\lambda_{i}^{4}=v_{r}-c_{s}$, where the $c_{f}\left(c_{s}\right)$ are the fast (slow) magnetosonic speed. $c_{f}=\frac{1}{2} \sqrt{v_{s}^{2}+v_{A}^{2}+\left(\left(v_{s}^{2}+v_{A}^{2}\right)^{2}-4 v_{s}^{2} \frac{B_{r}^{2}}{\mu \rho}\right)^{\frac{1}{2}}}, c_{s}=$ $\frac{1}{2} \sqrt{v_{s}^{2}+v_{A}^{2}-\left(\left(v_{s}^{2}+v_{A}^{2}\right)^{2}-4 v_{s}^{2} \frac{B_{r}^{2}}{\mu \rho}\right)^{\frac{1}{2}}}, \quad$ where $v_{s}=\sqrt{\frac{\gamma p}{\rho}}, v_{A}=$ $\sqrt{\frac{B_{r}^{2}+B_{\theta}^{2}+B_{\phi}^{2}}{\mu \rho}}$ are the sound and Alfvénic speeds. When the index 

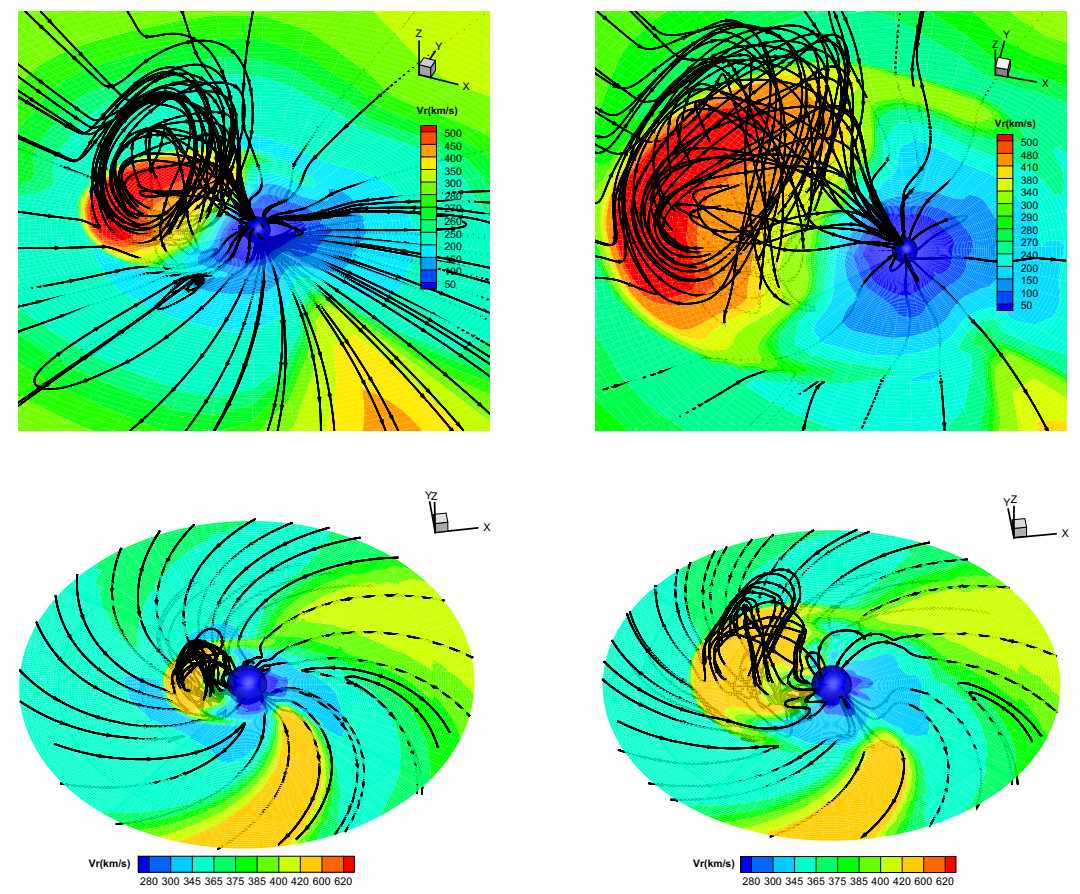

Fig. 4. A $3 \mathrm{D}$ representation of the CME is shown at $3 \mathrm{~h}$ (top left), $6 \mathrm{~h}$ (top right), $20 \mathrm{~h}$ (bottom left), and $40 \mathrm{~h}$ (bottom right). The color code represents the velocity magnitude in the $x-y$ equatorial plane. The streamlines showed in black denote the magnetic field lines. The blue sphere in the first row is the Sun, and in the bottom row represent $20 R_{s}$.

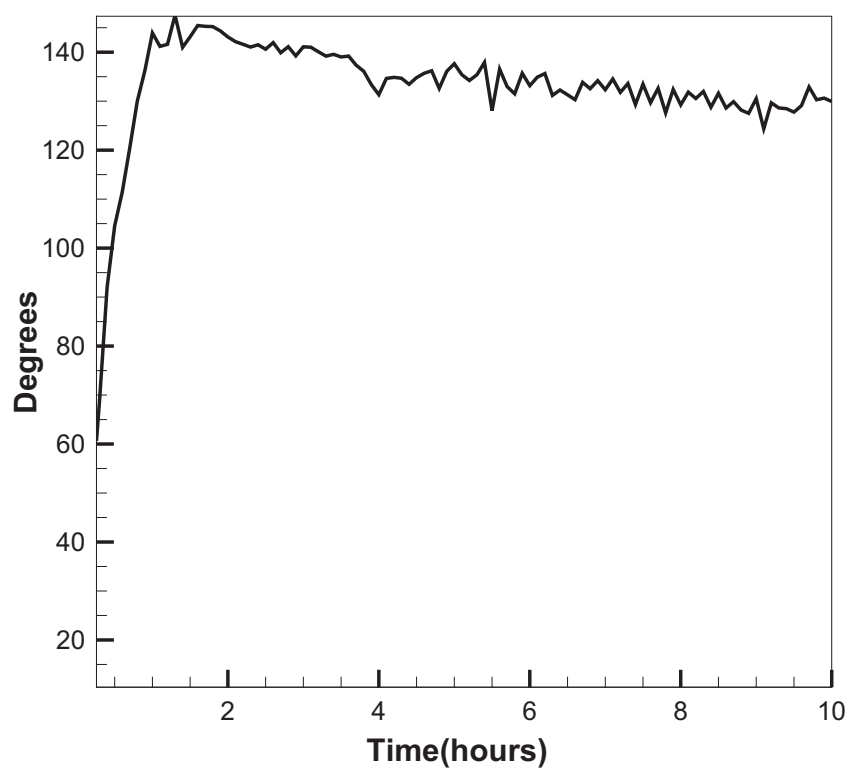

Fig. 5. The evolution of the spread angle of the CME in time.

$\lambda_{\max }$ is positive and large, the cell surface is close to the shock front. The shock is stronger when the index is larger. Here a shock front is clearly visible at $15 R_{s}$ for $t=3 \mathrm{~h}$, at $25 R_{s}$ for $t=6 \mathrm{~h}$. At the shock front, the solar wind plasma becomes compressed and heated. This is clearly visible according to the enhancements of velocity and relative density.
Figures 8 and 9 present the evolution of the relative density $\frac{\rho-\rho_{0}}{\rho_{0}}$ and magnetic fields $\frac{B}{B_{0}}$ versus heliocentric distance along $\theta=90^{\circ}, \phi=190^{\circ}$ and $\theta=90^{\circ}, \phi=180^{\circ}$ at different hours. $B$ is the total magnetic fields, and $B_{0}$ is the magnetic fields of background solar wind. At the early time of $t=3 \mathrm{~h}$ after CME onset, the relative density profile has an obvious sharp jump from $\sim 10 R_{s}$ to $\sim 15 R_{s}$. The width of the relative density jump increased apparently as the time gone. At $20 \mathrm{~h}$, the width of the jump is near $25 R_{s}$ and at $40 \mathrm{~h}$, the width increases to $40 R_{s}$. The evolution of the relative magnetic fields have a similarity time profiles. Thus, the CME expands in radial direction as it propagates into the heliosphere. From this figure, we also find the relative density behind the CME decreased after the CME passed, there is a rarefaction region behind the travelling plasma, which lowers the density, pressure, and speed of the wind immediately ahead of the CME.

Figure 10 shows the simulated profiles of the relative density $\frac{\rho-\rho_{0}}{\rho_{0}}$ in the $x-y$ equatorial plane at $3 \mathrm{~h}, 6 \mathrm{~h}, 20 \mathrm{~h}$ and $40 \mathrm{~h}$. The white circle in the first row is the Sun and represent $20 R_{s}$ in the bottom row. The top row shows the domain from $1 R_{S}$ to $20 R_{s}$, while the bottom row views from $20 R_{S}$ to $1 \mathrm{AU}$. From this figure, we find that the CME almost propagates along the Parker spiral and the CME is asymmetrical in heliolongitude because of the non-uniform solar wind ambient.

Figure 11 compare the model results with the Wind observations. Shown are number density, flow velocity, magnetic field, and three components of magnetic field in GSE coordinates respectively, from top to bottom. The simulated results at the Earth are shown by blue dot lines. The Wind observations 

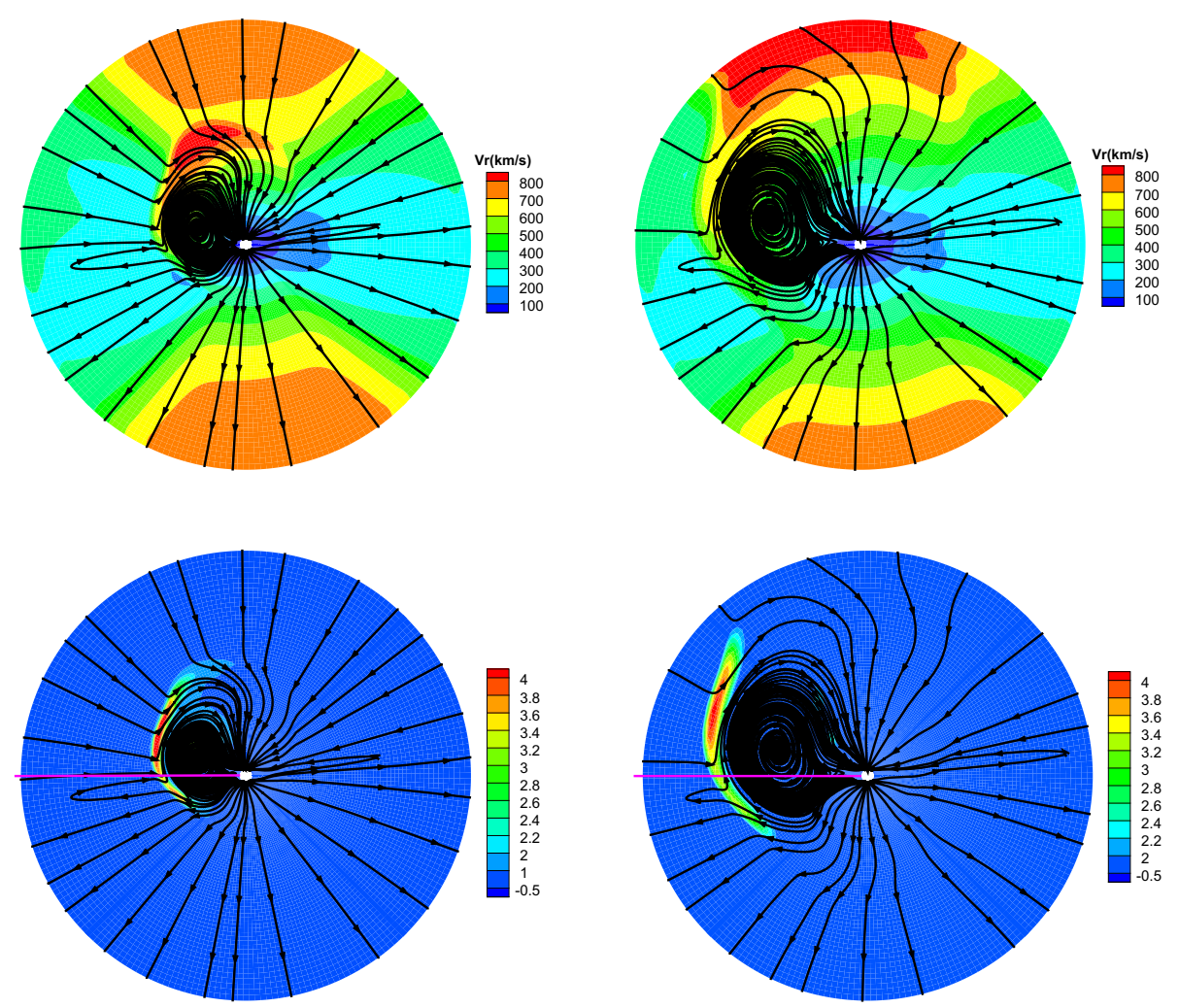

Fig. 6. Time evolution of the CME in the $x-z$ meridional plane at $3 \mathrm{~h}$ (left), $6 \mathrm{~h}$ (right). Solid black lines display the projection of the magnetic field lines, the false color images in the top row are velocity and in the bottom row are relative density.
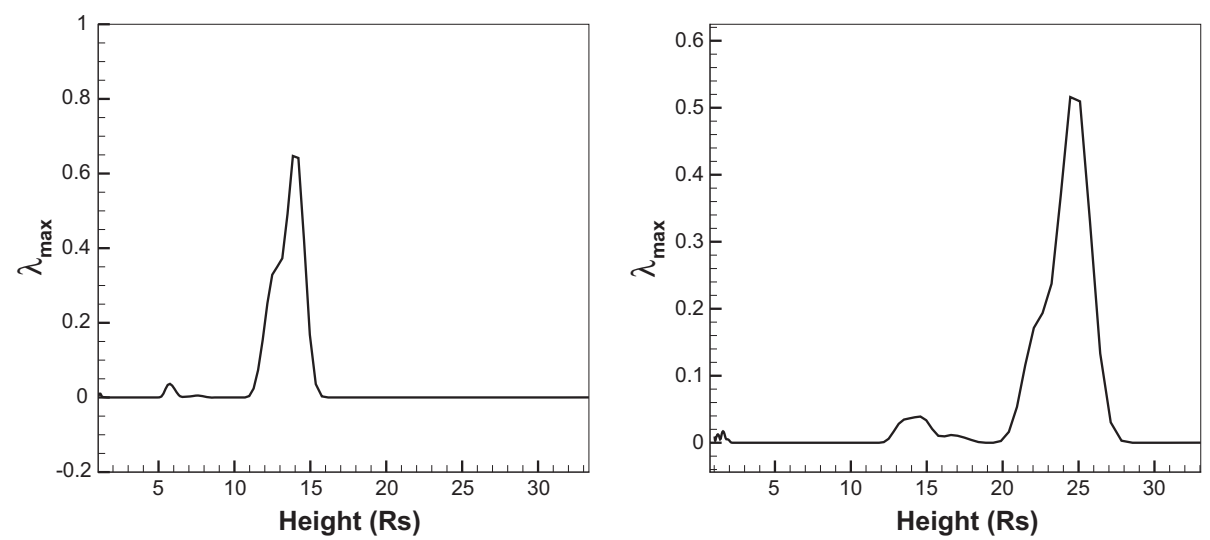

Fig. 7. Evolution of the spatial difference in the characteristic speed versus heliocentric distance.

are shown by black solid lines. As it can be seen from Figure 11, our simulation has reproduced some of the in situ measurements in spite of the simple CME model used in this paper; the general shape of the curves is similar, and the three components of magnetic field display realistic values. We find that the simulated background velocity and density at the Earth is higher. The simulated velocity is almost $150 \mathrm{~km} / \mathrm{s}$ greater than observation. The reason for this may be due to the single fluid model used and the volumetric heating assumption in our code, which has also been pointed out by (Feng et al., 2010; Zhou et al., 2012; Feng et al., 2014). We use a volumetric heating method based on the magnetic field topology to obtain a typical background solar wind in this paper. This heating form cannot be the only acceleration process acting on the solar wind and that other presently unknown sources are needed to act within the region between the lower corona and the source surface. Further characterizing and quantifying of the key physical processes/ mechanism will clarify an operational route to more physically integrate realistic coronal heating modules into 3D MHD codes (Feng et al., 2014). The arrival time of the CME at $1 \mathrm{AU}$ for the model results are earlier than observations. We find that the CME front arrives at Wind on 16 December at 07:20 UT. 
(a)

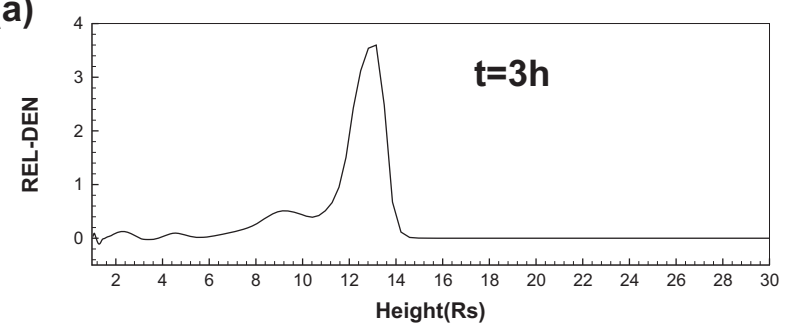

(c)

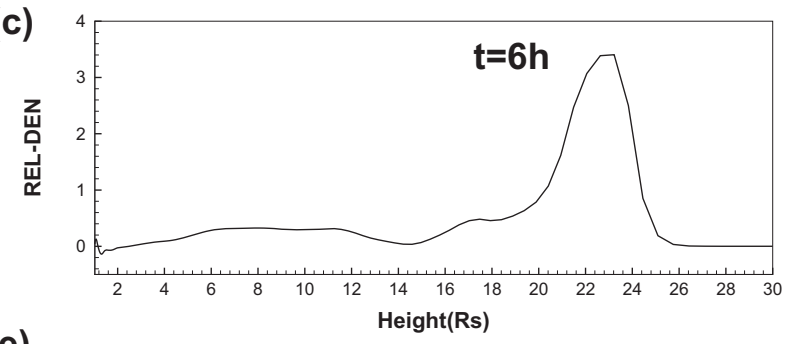

(e)
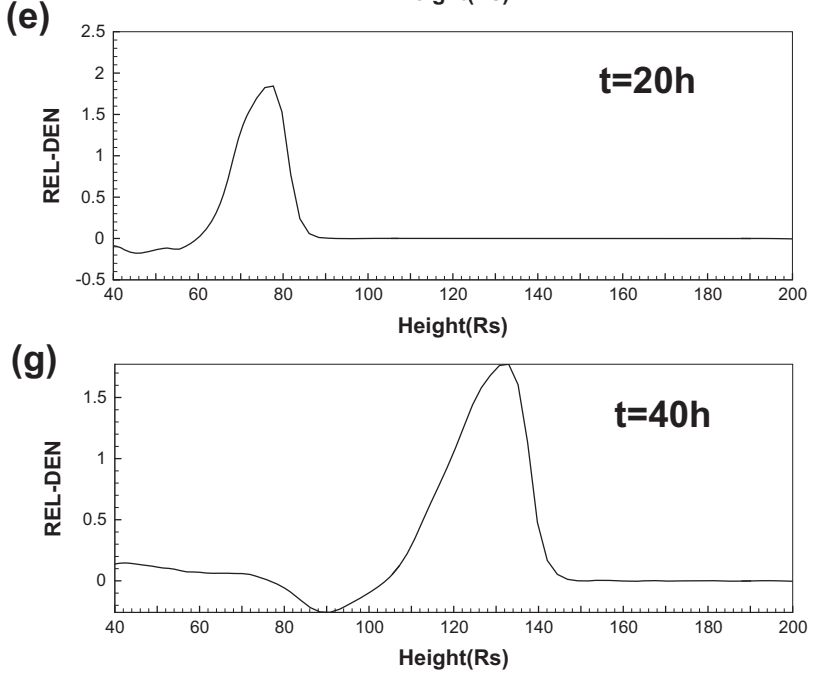

(b)
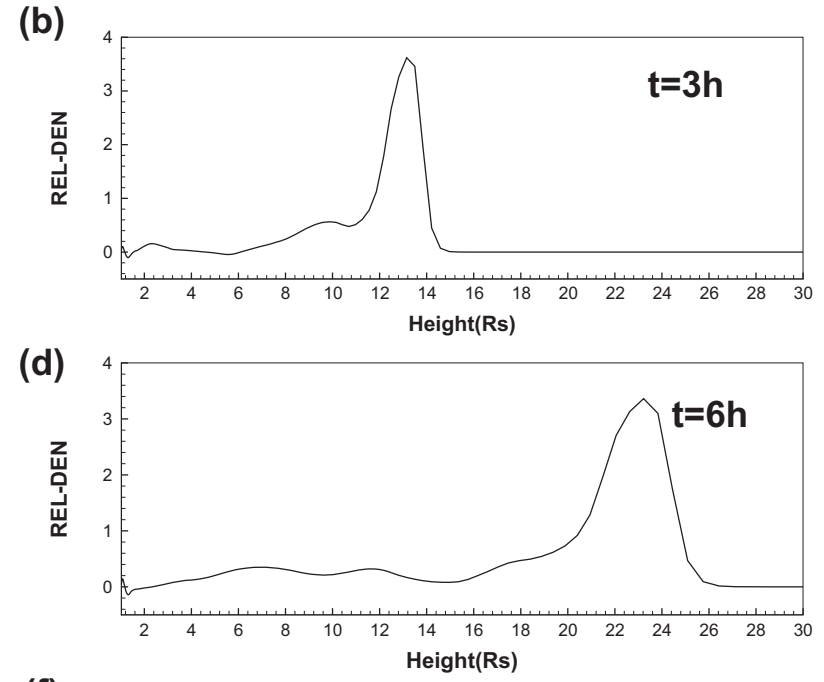

(f)

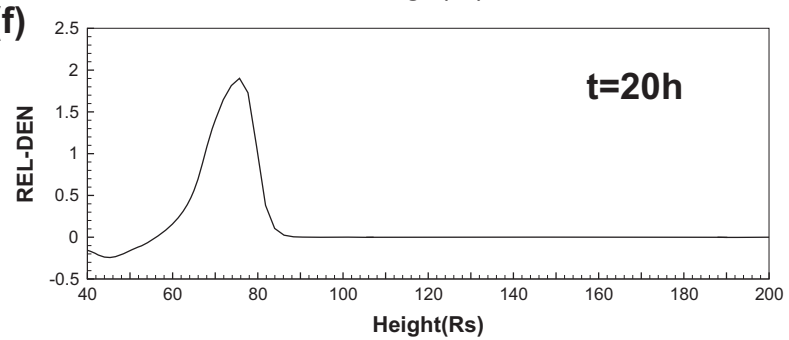

(h)

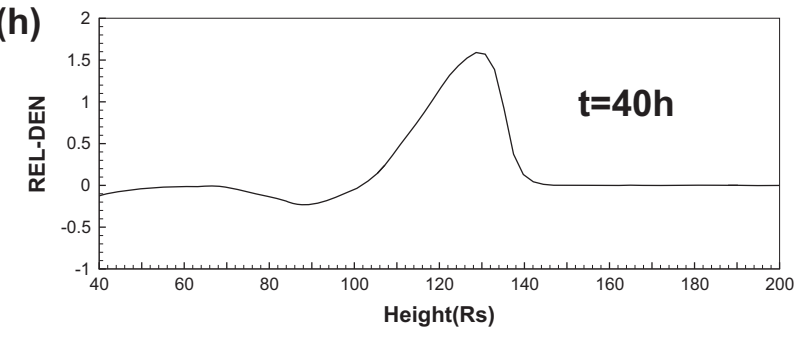

Fig. 8. Evolution of the relative density $\frac{\rho-\rho_{0}}{\rho_{0}}$ versus heliocentric distance along $\theta=90^{\circ}, \phi=190^{\circ}$ (left column) and $\theta=90^{\circ}, \phi=180^{\circ}$ (right column) at $t=3,6,20$, and $40 \mathrm{~h}$, respectively. The top two rows (a, b, c, d) show the domain from $1 R_{s}$ to $30 R_{s}$, while the bottom two rows (e, f, g, h) view from $40 R_{s}$ to $200 R_{s}$.

Our model predicts the CME arrival at Earth at about 00:05 UT on 16 December, with an error of $7 \mathrm{~h}$ compared to the Wind CME arrival time. For the magnetic field, it matches the observations well. $B_{x}$ and $B_{y}$ is decreased due to passage of the CME. The $z$ component of the magnetic field for simulated and measured profiles at the Earth are similar, becoming northward first, then changing to southward smoothly. Since the $B_{z}$ component is a key parameter to predict the intensity of a magnetic storm, it is important to capture some realistic features in simulation.

Figure 12 shows the evolution of the three components of magnetic field along heliocentric distance with different latitudes at $t=0 \mathrm{~h}$. The columns from left to right are $B_{x}, B_{y}$ and $B_{z}$ in GSE coordinates, respectively. $\Delta \theta=0^{\circ}$ means the Sun-to-Earth line with $(\theta, \phi)=\left(90^{\circ}, 180^{\circ}\right)$, and $\Delta \theta=-30^{\circ}$ represented the line in $r$ direction with $(\theta, \phi)=\left(60^{\circ}, 180^{\circ}\right)$. Since the observed CME is launched at N34 in our paper, the line for $\Delta \theta=-30^{\circ}$ represented the magnetic fields in CME while the line for $\Delta \theta=0^{\circ}$ represented the solar wind magnetic fields without disturbance. Passing the CME, $B_{x}$ and $B_{z}$ changed direction two times, first become negative and then positive. The $B_{y}$ changed direction three times, first positive, then negative and finally positive.

Figure 13 shows the evolution of the three components of magnetic field along heliocentric distance with different longitudes at $t=3,6,20$ and $60 \mathrm{~h}$ from top to bottom. $\Delta \phi=0^{\circ}$ means the Sun-to-Earth line with $(\theta, \phi)=\left(90^{\circ}, 180^{\circ}\right), \Delta \phi$ $=6^{\circ}$ (west of the Sun-to-Earth line) and $\Delta \phi=-6^{\circ}$ (east of the Sun-to-Earth line) represented the lines in $r$ direction with $(\theta, \phi)=\left(90^{\circ}, 186^{\circ}\right)$ and $(\theta, \phi)=\left(60^{\circ}, 174^{\circ}\right)$. At $t=3 \mathrm{~h}$ (first row), since the CME quickly expands, the three lines are effected by the CME. The general shape of the curves are similar for the three components of magnetic field. The directions in $B_{y}$ and $B_{z}$ agree with the initial magnetic fields in CME. The $B_{x}$ component became negative, which is different from the initial $B_{x}$ in CME, that is may be resulted by the interaction between $\mathrm{CME}$ and solar wind. At $t=6,20$ and $60 \mathrm{~h}$, the structures for the three components of magnetic field kept unchanged and are almost the same as the $t=3 \mathrm{~h}$. From Figures 12 and 13, we find 

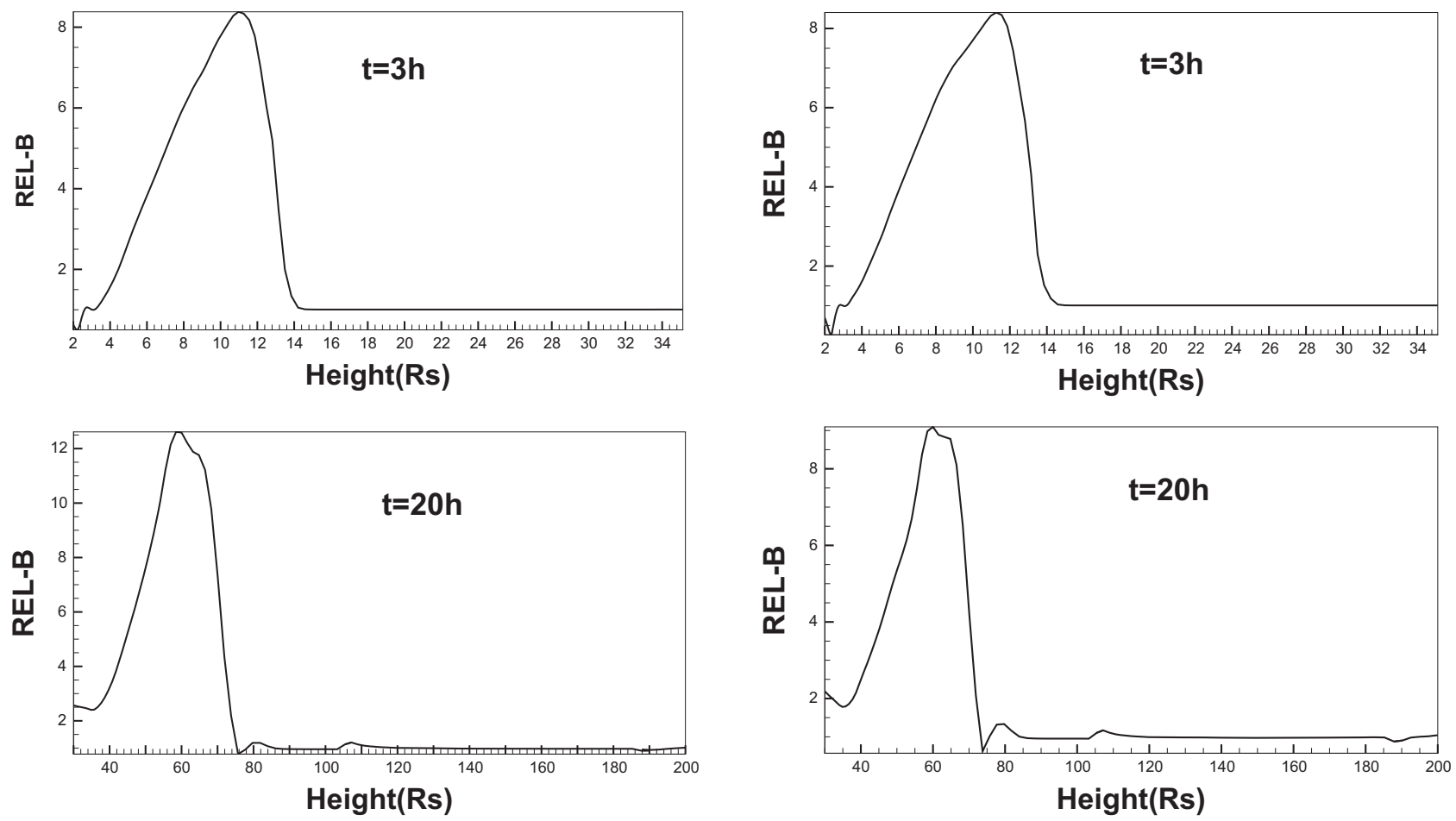

Fig. 9. Evolution of the relative magnetic fields $\frac{B}{B_{0}}$ versus heliocentric distance along $\theta=90^{\circ}, \phi=190^{\circ}$ (left column) and $\theta=90^{\circ}$, $\phi=180^{\circ}$ (right column) at $t=3$ and $20 \mathrm{~h}$, respectively. The top row shows the domain from $1 R_{s}$ to $35 R_{s}$, while the bottom row views from 30 $R_{s}$ to $200 R_{s}$.
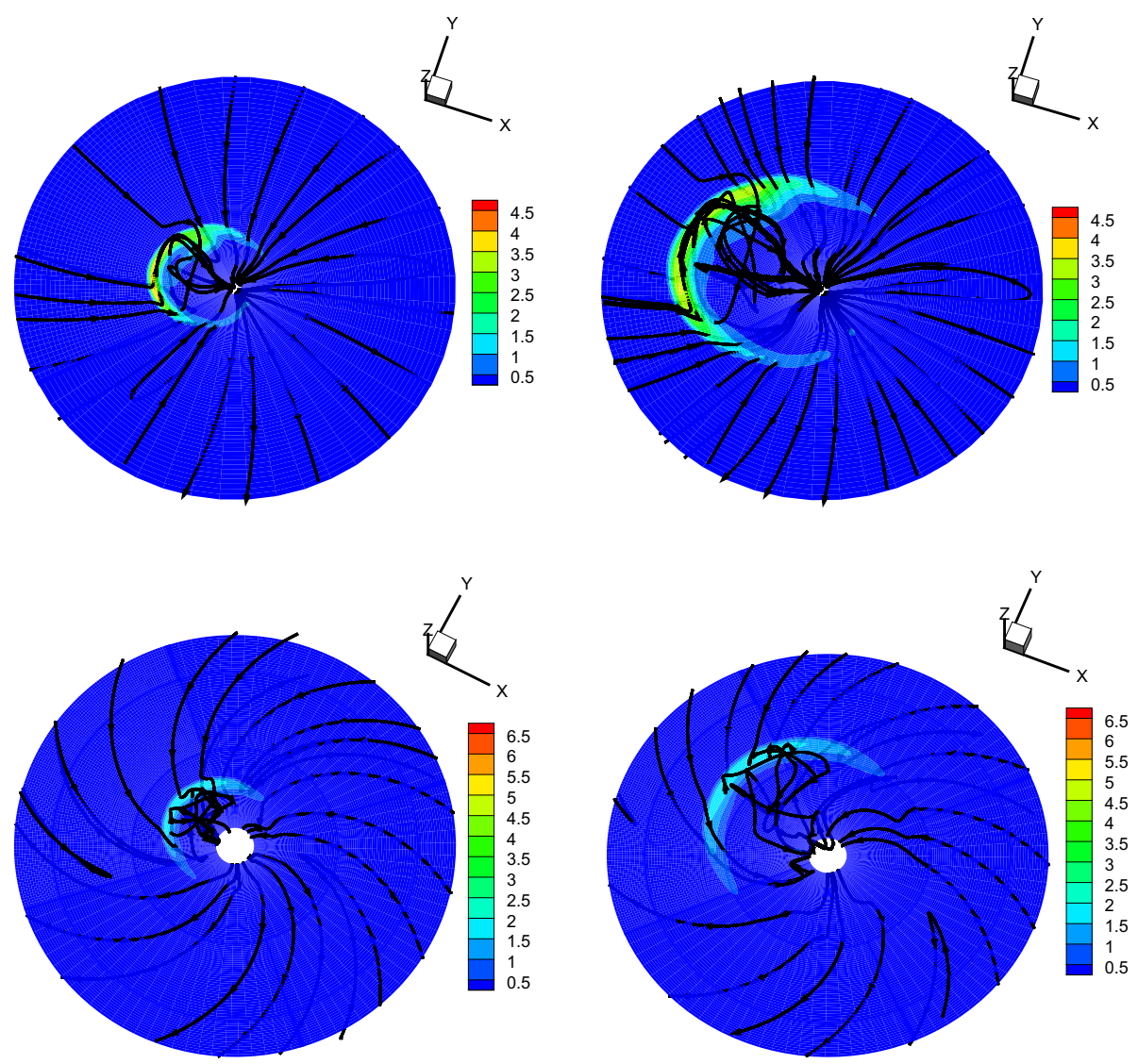

Fig. 10. The simulated profiles of the relative density in the $x-y$ equatorial plane at $3 \mathrm{~h}$ (top left), $6 \mathrm{~h}$ (top right), $20 \mathrm{~h}$ (bottom left), $40 \mathrm{~h}$ (bottom right). Solid black lines display magnetic streamlines. The write circle in the first row is the Sun, and in the bottom row represent $20 R_{s}$. 

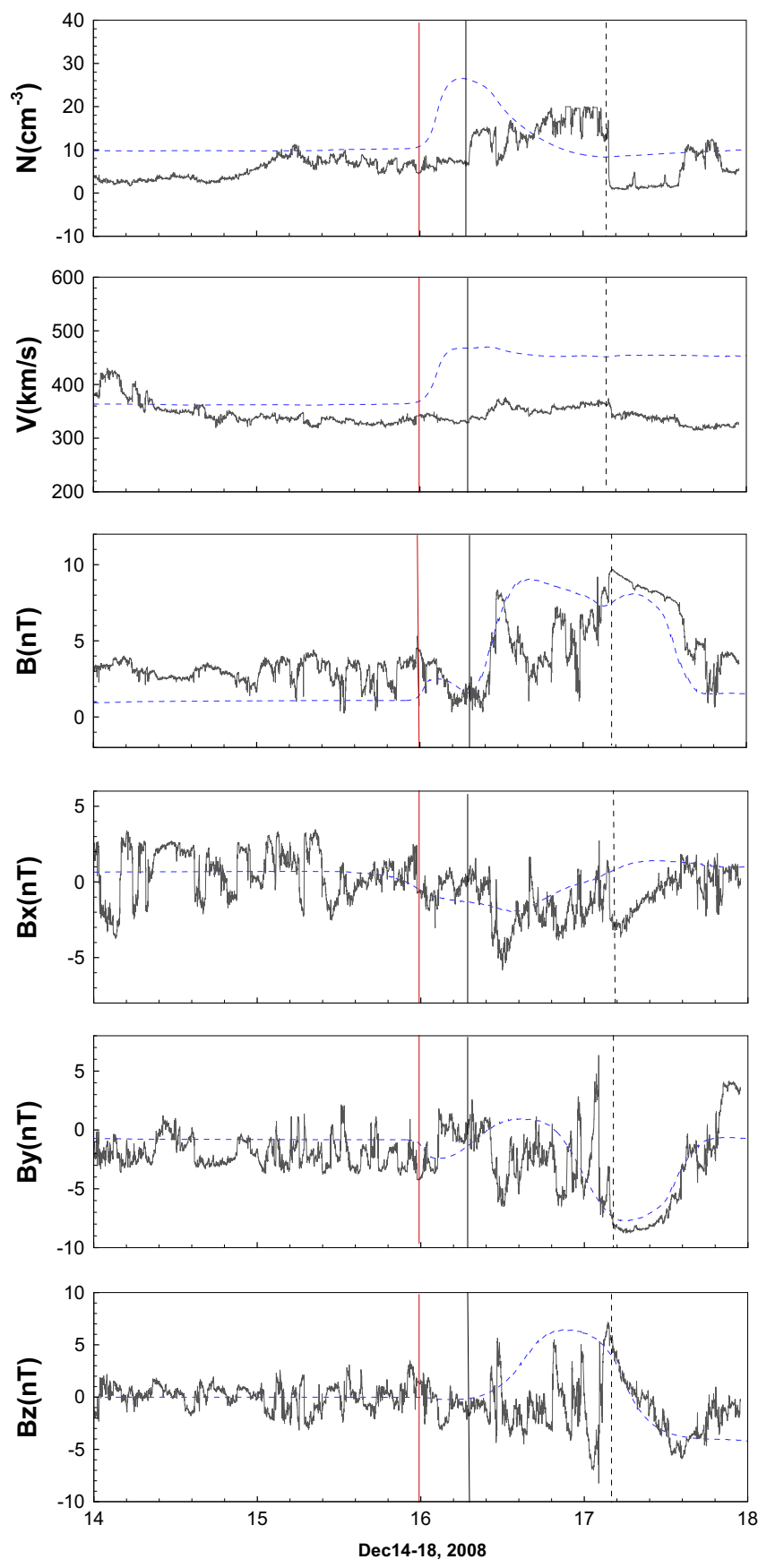

Fig. 11. Evolution of solar wind parameters at the Earth. Shown are number density, flow velocity, magnetic field, and three components of magnetic field in GSE coordinates. The simulated results at the Earth are shown by blue dot lines. The Wind observations are shown by black solid lines. The black vertical solid lines and dashed lines indicate the arrival time at $1 \mathrm{AU}$ of the CME front and the magnetic cloud from observations, respectively. The red vertical solid lines indicate the arrival time at $1 \mathrm{AU}$ of the $\mathrm{CME}$ front from model results.

the magnetic fields of the CME at $1 \mathrm{AU}$ have a large dependence on the initial magnetic fields, such as $B_{y}$ and $B_{z}$. The general structure of magnetic fields along Sun-Earth line kept almost unchanged after $3 \mathrm{~h}$, thus, we can conclude the general structure of magnetic fields at $1 \mathrm{AU}$ just after $3 \mathrm{~h}$ of the simulation began.

\subsection{Numerical results assuming different parameters of the CME}

In this section, we showed the results at $1 \mathrm{AU}$ with different parameters of the CME. We changed one parameter every time, and the other parameters remained the same as above. Firstly, we compared the results for which the initial speeds for CME $V_{\mathrm{cme}}$ are varied. Figure 14 shows the evolution of solar wind parameters at the Earth with different velocities $(200 \mathrm{~km} / \mathrm{s}$, $300 \mathrm{~km} / \mathrm{s}$, and $400 \mathrm{~km} / \mathrm{s}$ ) in the CME. The number density for the three results are similar. The velocity have some differences, and CME with higher initial velocity would result higher velocity at $1 \mathrm{AU}$. The magnetic fields have little difference for the three cases.

As we know, the evolution of CMEs is mainly governed by the Lorentz and the aerodynamic drag force. In this paper, the very limited effect of the CME's speeds on the final conditions suggests the plasma acceleration in the wind is mostly due to the drag force by the background. CMEs which are faster than the ambient solar wind are decelerated, whereas those slower than solar wind are accelerated by the ambient flow. For these CMEs with larger momentum, the plasma is pulled and decelerated under more drag force, which possibly comes from the slow solar wind or the front shock wave structure in the interplanetary space.

Figure 15 presented the evolution of solar wind parameters at the Earth with different magnitude of magnetic fields $B_{\text {cme }}$ in the CME. We choose $B_{\text {cme }}=5$ Gauss, 4 Gauss, and 3 Gauss. From this figure, we found the magnetic fields magnitude $B_{\text {cme }}$ had a large effect on the model results. The arrival time at Earth for $B_{\mathrm{cme}}=5$ Gauss is earliest, and for $B_{\mathrm{cme}}=3$ Gauss is latest. The CME with higher magnetic fields magnitude would result higher solar wind parameters at the Earth, which can be seen from the number density, flow velocity, and magnetic field in this figure.

The reason for this dissemblance must be due to the differences in the initial magnetic pressure since the initial magnetic field is the only unlikeness in the three models. At the Sun, white light observations suggest that CMEs are undergoing strong expansion because of a higher internal pressure (Riley \& Crooker, 2004). The CME with higher magnetic fields magnitude would result a higher pressure gradient between the flux rope and the background solar wind. So, the expansion speed will be higher for the higher magnetic fields magnitude in the CME (Riley \& Crooker, 2004; Byrne et al., 2010). There are many authors investigated the empirical relationship between the radial speed and the expansion speed of CMEs (Lago et al., 2003; Gopalswamy et al., 2009, 2012; Mäkelä et al., 2016). They found a high correlation between these two speeds. A CME with higher expansion speed will also have higher radial speed. So the $B_{\text {cme }}=5$ Gauss arrives earlier, this results is in agreement with the previous studies.

For the simulation of the 12 December 2008 CME in Section 5.1, the direction of the magnetic field is clockwise in the $x-z$ meridional plane, we referred this case as case 1 . Then, we changed the direction of the magnetic field and the magnetic field is anticlockwise in the $x-z$ meridional plane, we referred this case as case 2. Figure 16 show the evolution of solar wind parameters at the Earth with the two cases. Compared with case 1 , the arrival time at Earth for case 2 is later. The number density and flow velocity at Earth are also lower for case 2 . 

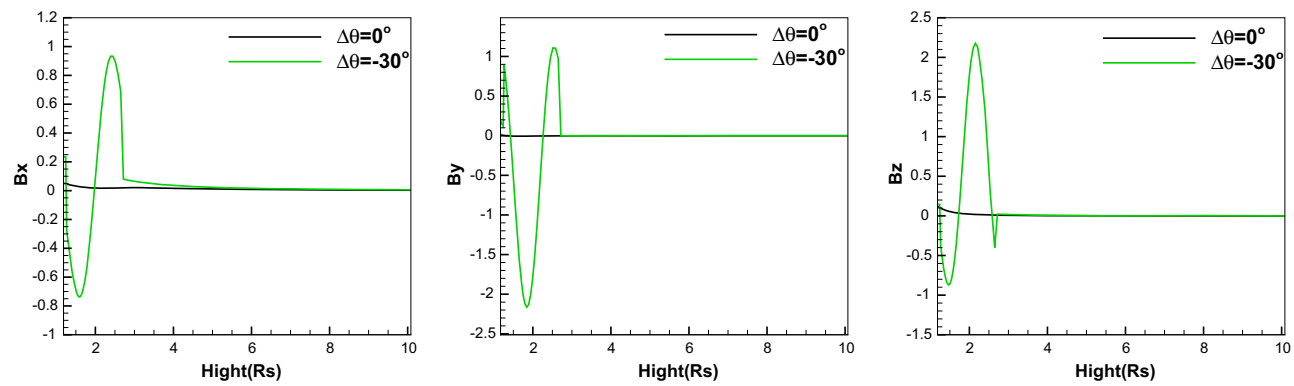

Fig. 12. Evolution of the three components of magnetic field along heliocentric distance with different latitudes at $t=0$. The columns from left to right are $B_{x}, B_{y}$ and $B_{z}$ in GSE coordinates, respectively.
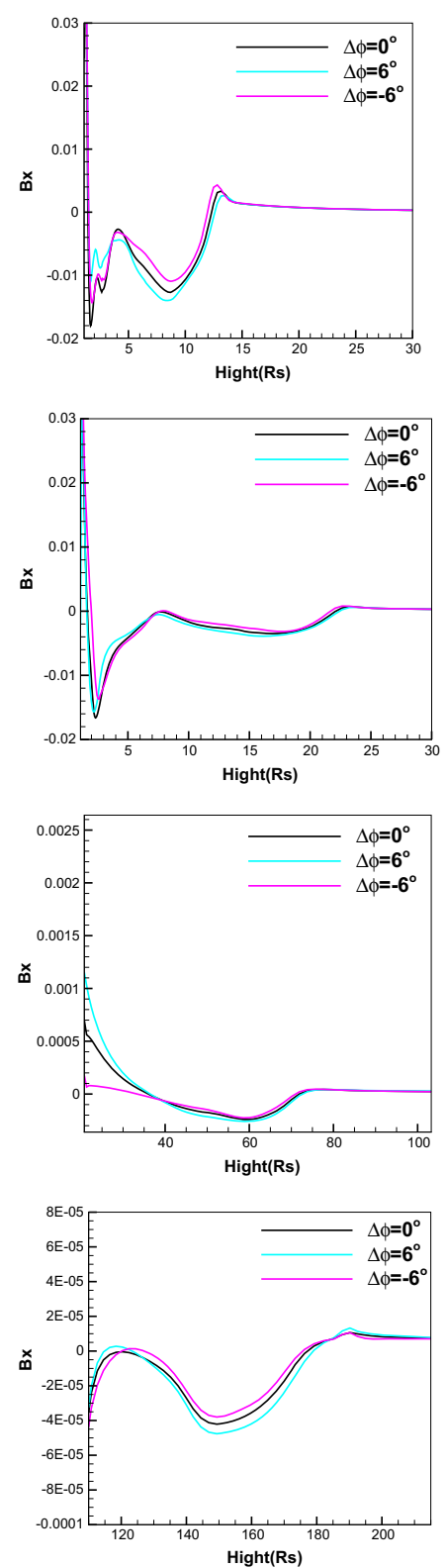
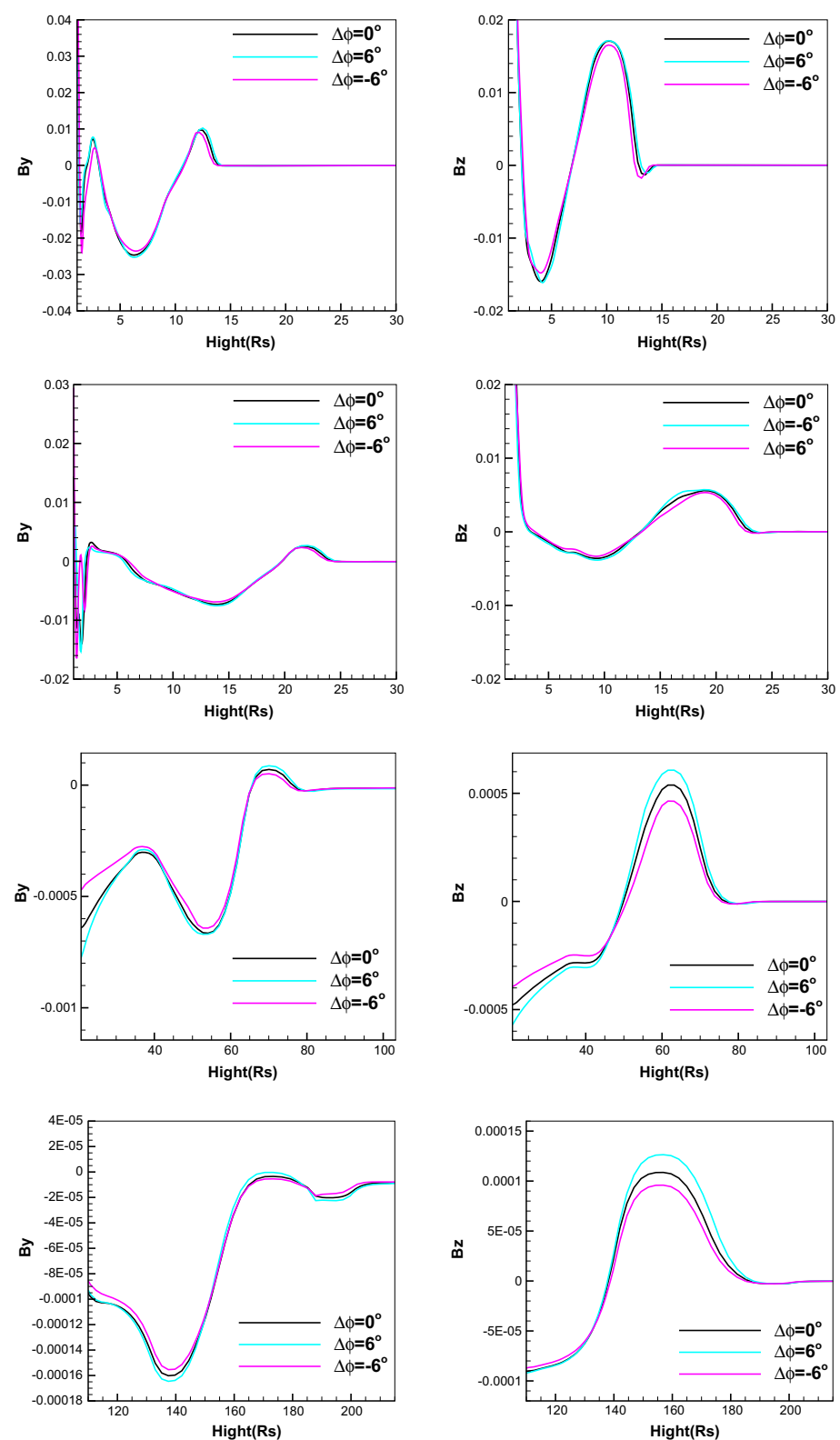

Fig. 13. Evolution of the three components of magnetic field along heliocentric distance with different longitudes at several times. The columns from left to right are $B_{x}, B_{y}$ and $B_{z}$ in GSE coordinates, respectively. The rows (top to bottom) are $t=3,6,20$ and $60 \mathrm{~h}$. 

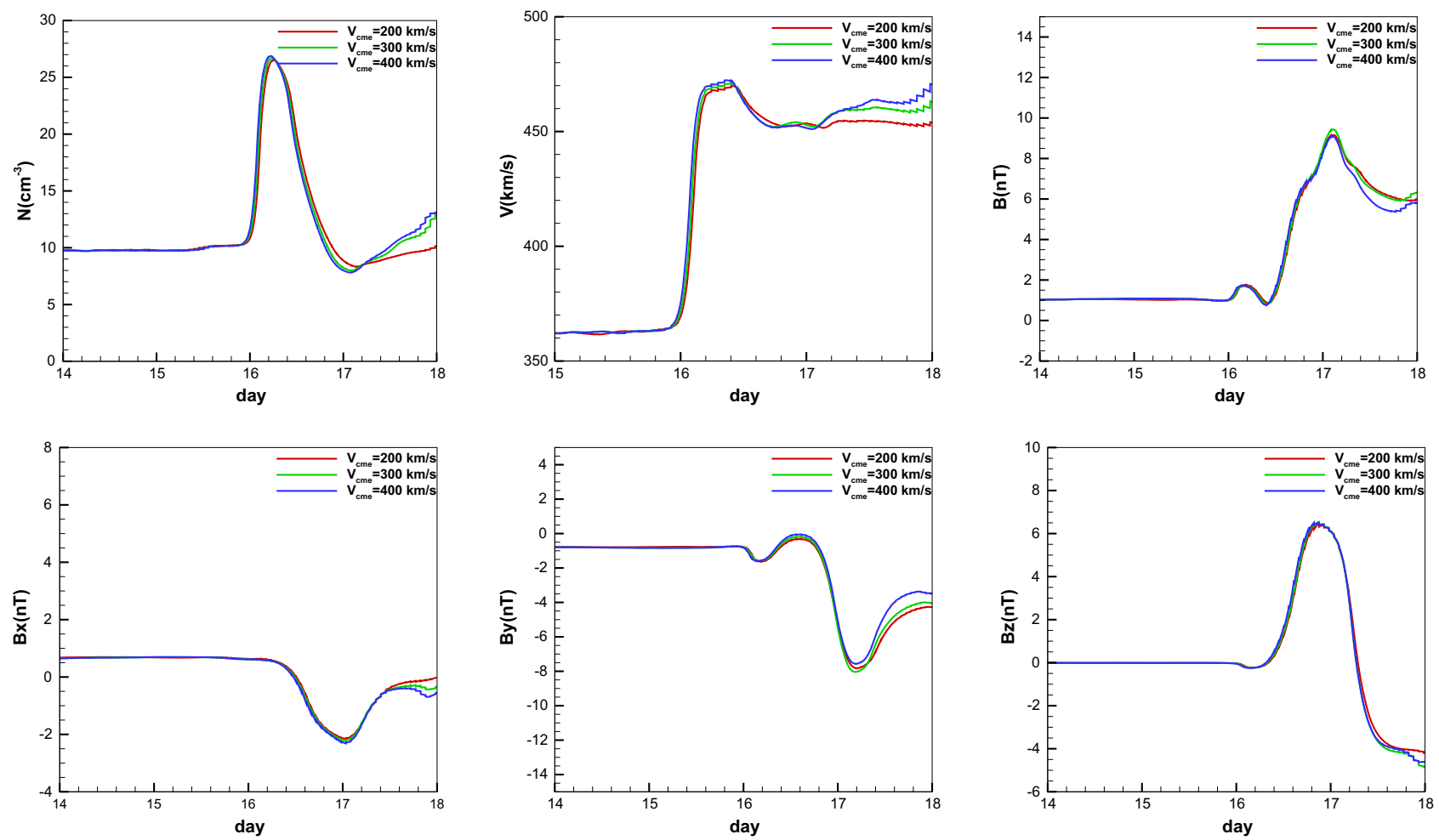

Fig. 14. Evolution of solar wind parameters at the Earth with different velocities in the CME. The first row present the number density (left), flow velocity (middle), magnetic field (right), and the second row are the three components of magnetic field $\left(B_{x}, B_{y}, B_{z}\right)$ in GSE coordinates.
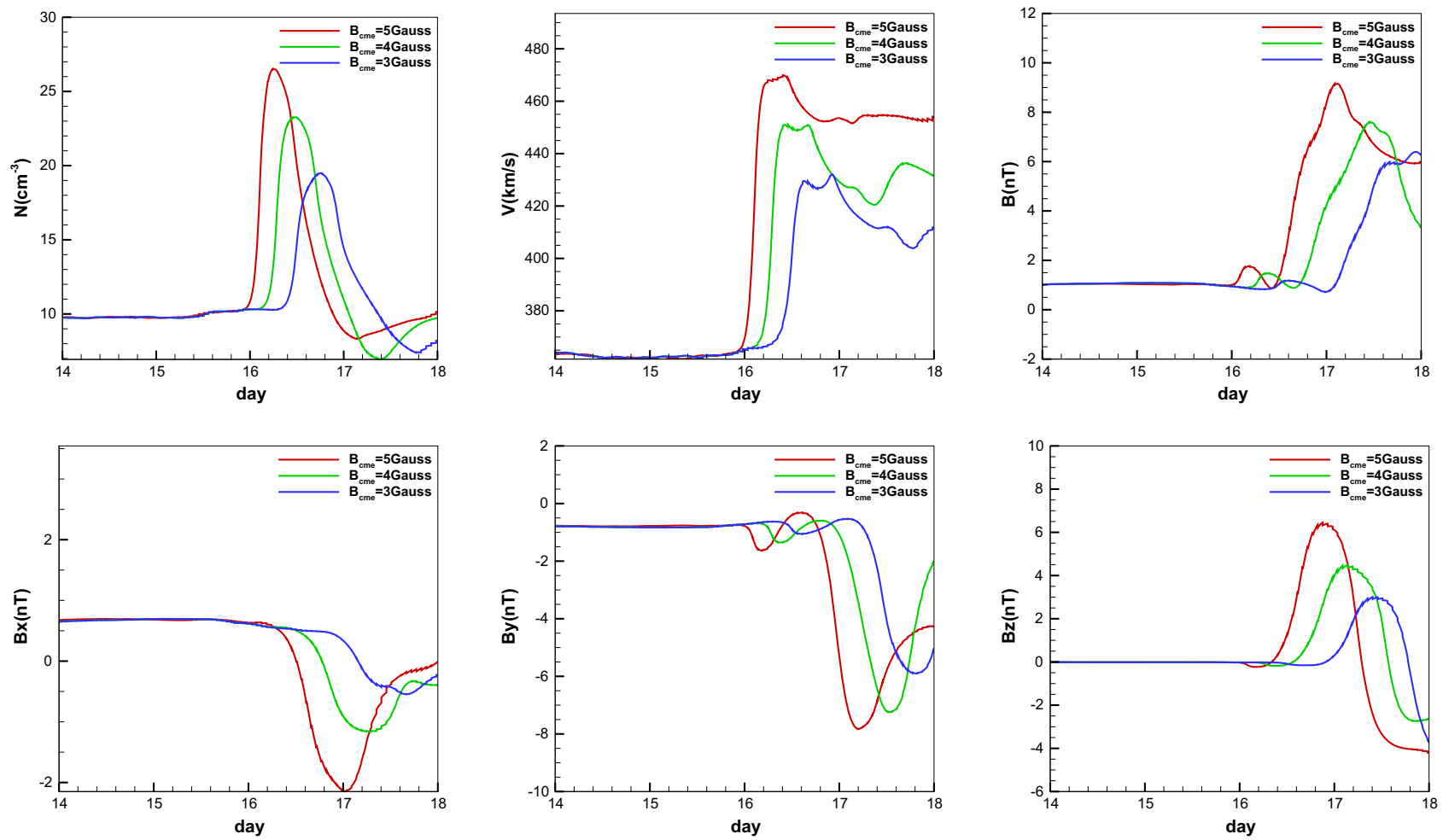

Fig. 15. Evolution of solar wind parameters at the Earth with different magnitude of magnetic fields in the CME. The first row present the number density (left), flow velocity (middle), magnetic field (right), and the second row are the three components of magnetic field $\left(B_{x}, B_{y}, B_{z}\right.$ ) in GSE coordinates. 

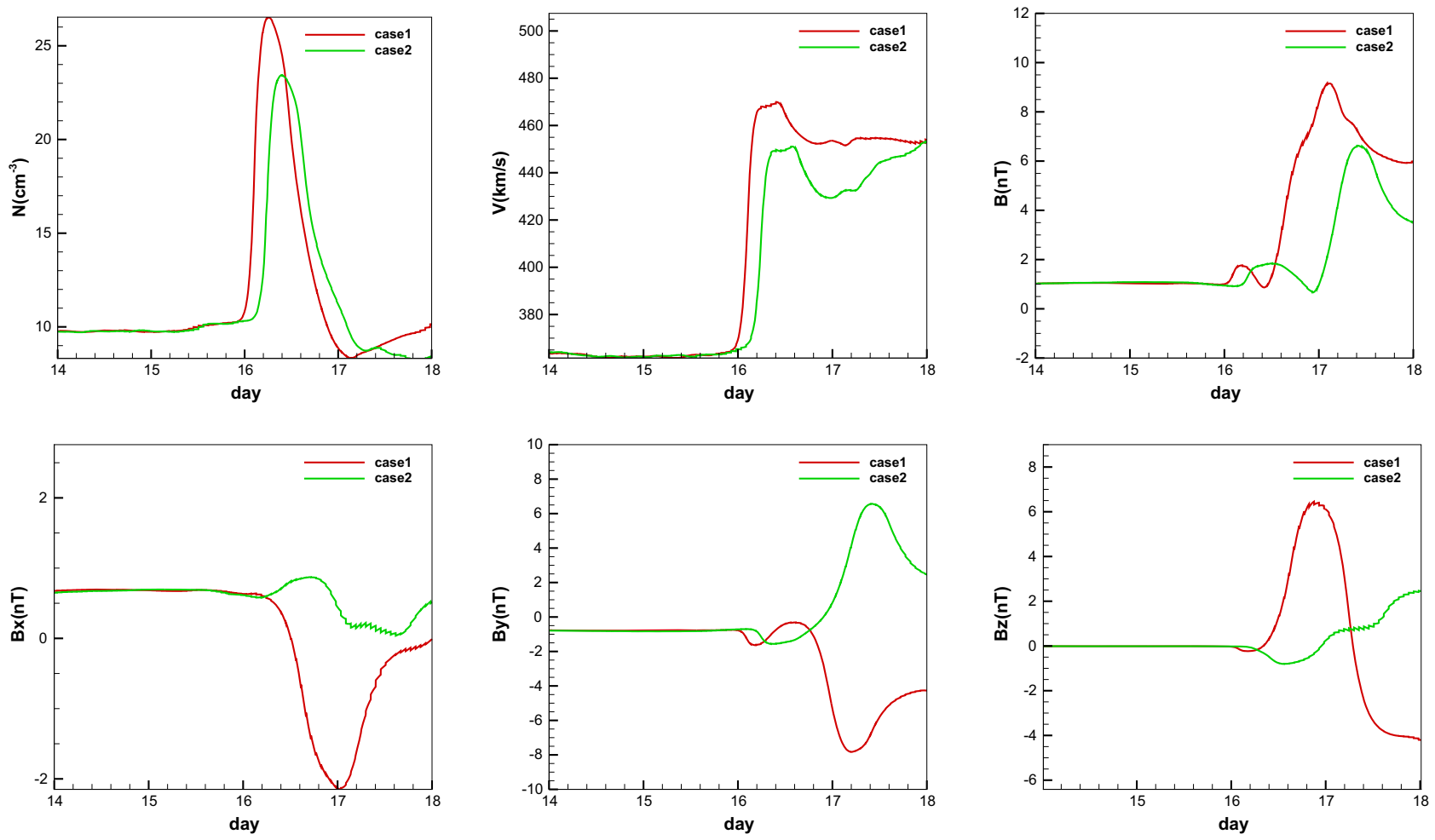

Fig. 16. Evolution of solar wind parameters at the Earth with different magnetic field direction in the CME. The first row present the number density (left), flow velocity (middle), magnetic field (right), and the second row are the three components of magnetic field ( $\left.B_{x}, B_{y}, B_{z}\right)$ in GSE coordinates.
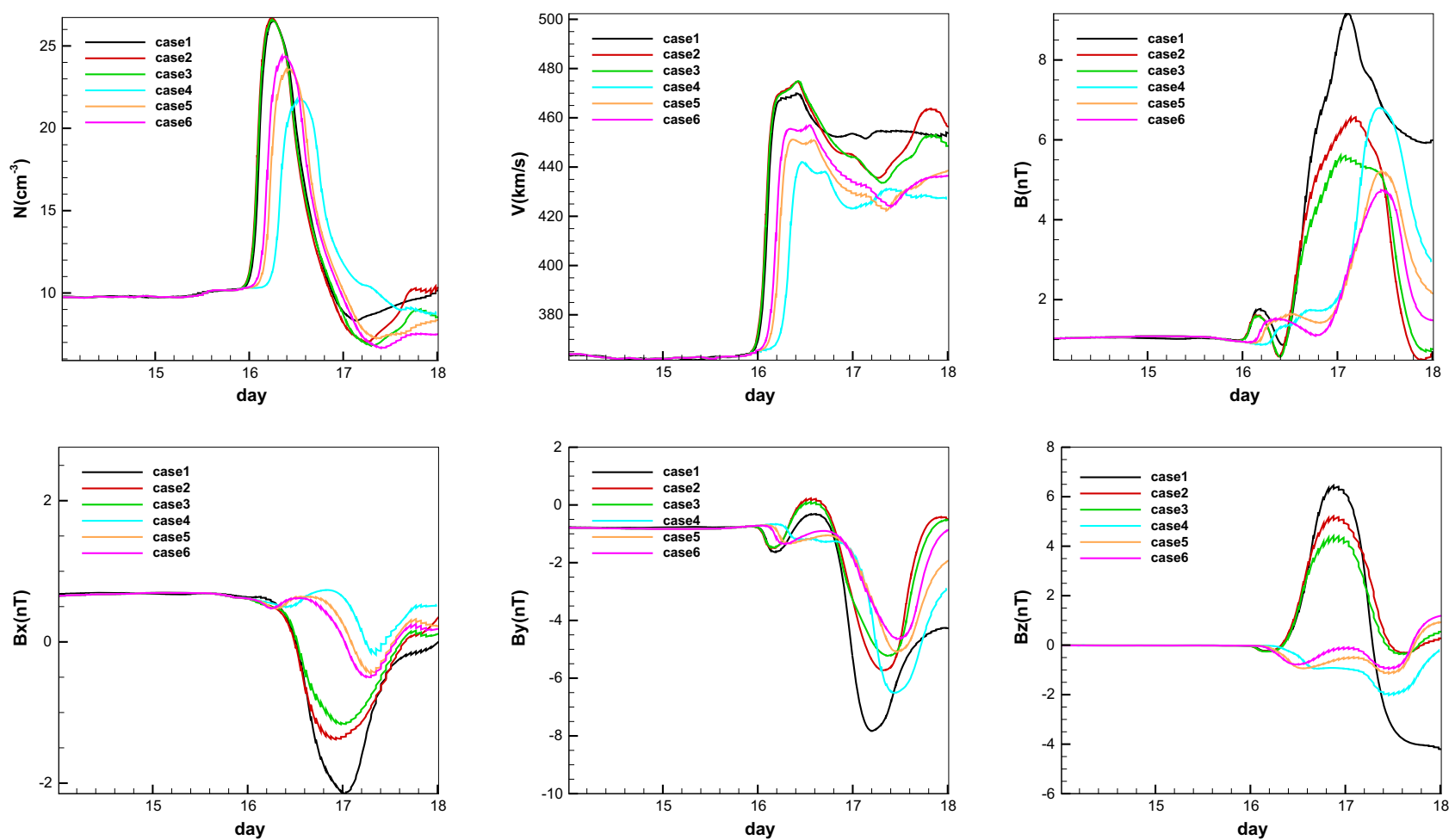

Fig. 17. Evolution of solar wind parameters at the Earth with different inclination angle of polar axis of CME. The first row present the number density (left), flow velocity (middle), magnetic field (right), and the second row are the three components of magnetic field ( $\left.B_{x}, B_{y}, B_{z}\right)$ in GSE coordinates. 

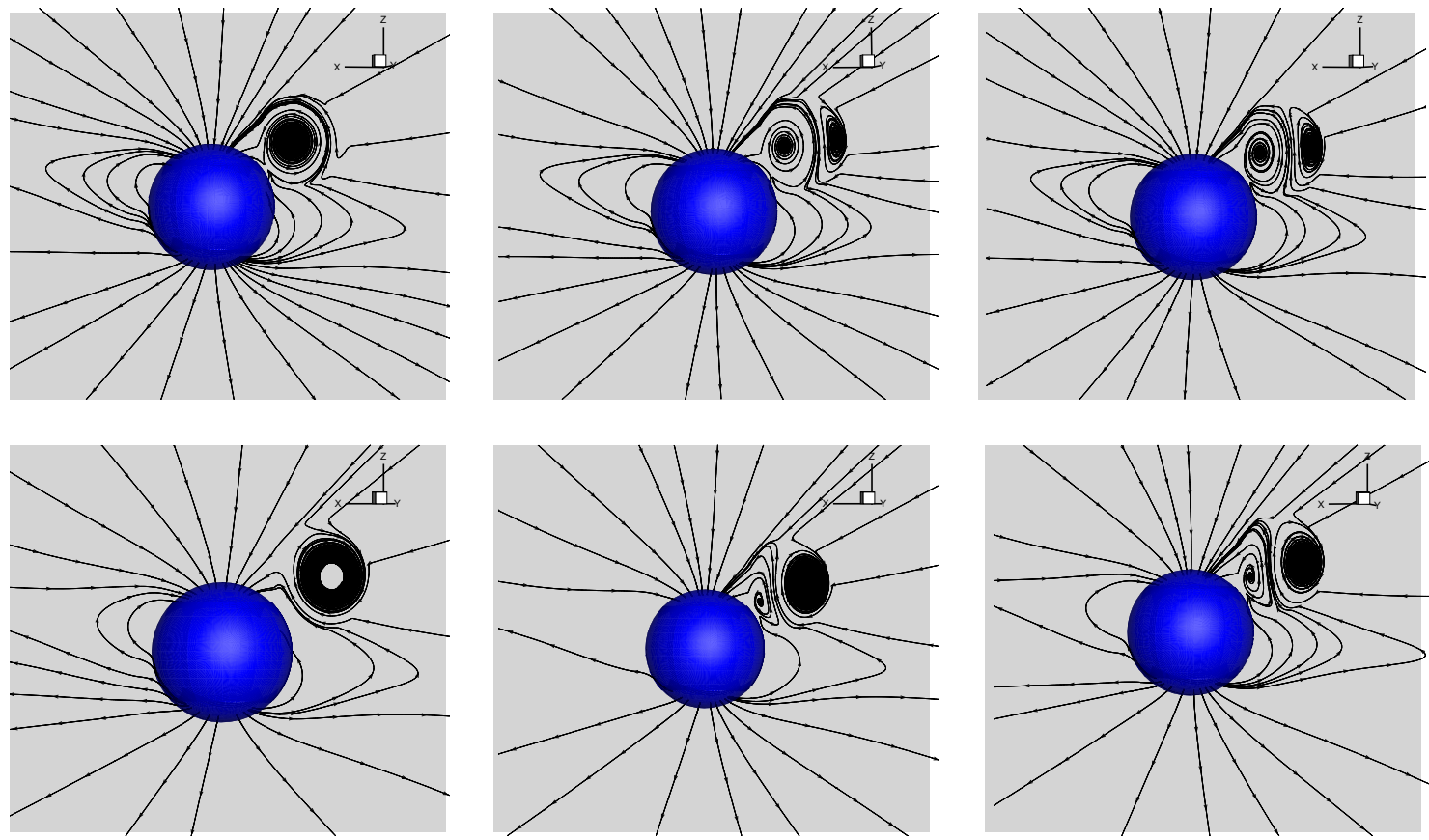

Fig. 18. The projected magnetic field lines in the $x-z$ meridional plane with different inclination angle of CMEs. The top row is for case 1 (left), case 2 (middle), case 3 (right), and the bottom is for case 4 (left), case 5 (middle), case 6 (right).

The three components of magnetic field are presented large differences for the two cases, especially for $B_{y}$. The $B_{x}$ and $B_{z}$ is lower for case 2, that is because the reconnection between the $\mathrm{CME}$ and solar wind takes place in case 2 since they have antiparallel magnetic lines.

Chané et al. $(2005,2006)$ also investigated the initial magnetic polarity on the evolution of CMEs. They have shown that the initial magnetic polarity of the CME flux rope affects its evolution path substantially: normal CMEs (the magnetic field of the CME has initially the opposite polarity compared to the background magnetic field) are deviated towards the pole while inverse CMEs (the magnetic field of the CME has initially the same polarity as the magnetic field of the background solar wind) are deviated towards the equatorial plane. The probability of hitting the Earth should be higher for inverse CMEs. Case 1 refers to inverse CME and Case 2 refers to normal CME. Since the observed CME is launched at N34 in our paper, the CME for case 2 are deviated towards the pole and we can only observed the flank at the Earth. Also, the reconnection takes place in case 2 remove the flux rope of the CME, so the magnetic field is lower for case 2 .

We changed the inclination angle of polar axis for the CME, and compared the results at $1 \mathrm{AU}$. We defined the inclination angle as $\Delta \tau$. The six cases are defined as follows: case 1 with $\Delta \tau=150^{\circ}$, case 2 with $\Delta \tau=120^{\circ}$, case 3 with $\Delta \tau=110^{\circ}$, case 4 with $\Delta \tau=30^{\circ}$, case 5 with $\Delta \tau=60^{\circ}$, case 6 with $\Delta \tau=70^{\circ}$. The inclination angle for case 1 - case 3 is larger than $90^{\circ}$, and the inclination angle for case $4-$ case 6 is smaller than $90^{\circ}$. Figure 17 shows the evolution of solar wind parameters at the Earth with different inclination angle of polar axis of CME. For the $B z$ component, the case $1-$ case 3 are better than case 2 - case 4 . Thus, the inclination angle larger than $90^{\circ}$ is reasonable for our simulation. For case 1 - case 3 , the number density and flow velocity are almost the same. The direction in the three components of magnetic field are similar for case 1 - case 3 , but the magnitude is different. The magnetic field will become lager if the inclination angle increased.

Figure 18 shows the projected magnetic field lines in the $x-z$ meridional plane with the six cases initially. For the case 4 with $\Delta \tau=30^{\circ}$, the magnetic field lines in the CME are antiparallel with the solar wind. The CME will deviate towards the pole during its outward propagation. When we increase the inclination angle, the part of the CMEs with antiparallel magnetic lines with solar wind will decrease. For case 1, the magnetic field lines in the CME become parallel with the solar wind. According to (Chané et al., 2005, 2006), CMEs have antiparallel magnetic lines with solar wind deviated towards the pole, while CMEs have parallel magnetic lines with solar wind deviated towards the equatorial plane. For case 4 - case 6 , the larger part of the CME have antiparallel magnetic lines with solar wind, so they may deviated towards the pole. Also, the reconnection between the CME and solar wind takes place, which remove the flux rope of the CME, so the magnetic field is lower for case 4 - case 6 . For case 1 - case 3 , the larger part of the CME have parallel magnetic lines with solar wind, so they may deviated towards the equatorial plane. The reconnection rate will become lower increasing the inclination angle, so the magnitude of magnetic field is lager for case 1 .

\section{Conclusions and discussions}

In this paper, we used a 3D MHD numerical model to simulate the propagation of a CME that occurred on 12 December 2008 from Sun to Earth in this paper. The 3D MHD numerical 
model is integrated in a finite-volume framework by splitting the MHD equations into two parts: fluid and magnetic induction (Feng et al., 2014), and the CT method is used to maintain the divergence constraint on the magnetic field.

To initiate the CME, we adopted a very simple 3D spherical plasmoid model which is superposed on the background solar wind, and the model has a high speed, density and pressure compared to the solar wind ambient (Kataoka et al., 2009; Zhou et al., 2012; Zhou \& Feng, 2013). The CME contains a spheromak-type magnetic field which is a linear force-free field in a completely isolated sphere. We use observations to determine the CME's position, and adjusted the CME initial parameters (density, magnetic field, velocity, and pressure) in the numerical simulation to yield a best fit with observations.

We then investigated the dynamical interaction between CME and background solar wind flow from Sun to 1 AU. After the CME onset, the magnetic field evolves nearly in self-similar manner. The plasmoid quickly expands because of the higher plasma density, speed, and magnetic pressure inside the CME. The radial and angular sizes of the CME increase with time, and the relative density behind the CME decreased after the CME passed. A shock front is clearly visible preceding the flux rope. At the shock front, the solar wind plasma becomes compressed and heated. The CME almost propagates along the Parker spiral and the CME is asymmetrical in heliolongitude because of the non-uniform solar wind ambient. We also compare the model results with the Wind observations. Our simulation has reproduced some of the in situ measurements in spite of the simple CME model used in this paper; the general shape of the curves is similar, and the three components of magnetic field display realistic values. The $B_{z}$ component become northward first, then changing to southward smoothly, this agree well with Wind observation. Since the $B_{z}$ component is a key parameter to predict the intensity of a magnetic storm, it is important to capture this realistic features for MHD models. Overall, our model provide a relatively satisfactory comparison with the Wind spacecraft observations at $1 \mathrm{AU}$, also many unsatisfactory points remain as discussed below.

We find that the simulated velocity and density are higher compared with Wind spacecraft. The simulated velocity is almost $150 \mathrm{~km} / \mathrm{s}$ greater than observation. The reason for this may be due to the volumetric heating assumption in our code. We use a volumetric heating method based on the magnetic field topology to obtain a typical background solar wind in this paper. This heating form cannot be the only acceleration process acting on the solar wind and that other presently unknown sources are needed to act within the region between the lower corona and the source surface. Further work should put emphasis on coronal heating modules, and take more physically integrate realistic coronal heating modules into 3D MHD codes.

We also investigated the numerical results assuming different parameters of the CME. Firstly, we compared the results for which the initial speeds for $\mathrm{CME}$ are varied. The number density and magnetic fields at $1 \mathrm{AU}$ are similar with different velocities, the velocities are higher at $1 \mathrm{AU}$ if the initial velocity in CME are higher. Then, we compared the solar wind parameters at the Earth with different magnitude of magnetic fields in the CME. We found the magnetic fields magnitude effected the arrival time of CME, and the arrival time is earlier if the initial magnetic fields in $\mathrm{CME}$ is larger. Also, the CME with higher magnetic fields magnitude would result higher solar wind parameters at the Earth. Next, we changed the direction of the magnetic field and the magnetic field is anticlockwise in the $x-z$ meridional plane. The three components of magnetic field presented large differences, especially for $B_{y}$. The $B_{x}$ and $B_{z}$ became lower, that is because the reconnection between the CME and solar wind takes place since they have antiparallel magnetic lines. Finally, the model results with different inclination angle of polar axis are compared at $1 \mathrm{AU}$. We found the inclination angle larger than $90^{\circ}$ is reasonable for our simulation. The direction in the three components of magnetic field are similar, but the magnitude are different. The magnetic will become lager if the inclination angle decreased. So the magnetic fields direction at $1 \mathrm{AU}$ are dependent on the inclination angle of polar axis in CME. Overall, the initial magnetic fields in the CME have a larger influence on the solar wind parameters at the Earth. The magnitude of initial magnetic fields in the CME will effect the the arrival time of interplanetary $\mathrm{CME}$, they also have a influernce on the magnitude of solar wind parameters at Earth. The initial direction of the magnetic field in CME have a larger influence on the structure of the interplanetary magnetic fields. These results are helpful in understanding and identifying the dynamic process occurring between the CME and the soalr wind.

Acknowledgements. The work is jointly supported by the National Natural Science Foundation of China (Grant Nos. 41731067, 41531073, 41504132, 41874202, and 41861164026) and the Specialized Research Fund for State Key Laboratories. The work was carried out at National Supercomputer Center in Tianjin, China and the calculations were performed on TianHe-1 (A). The editor thanks two anonymous referees for their assistance in evaluating this paper

\section{References}

Byrne JP, Maloney SA, Mcateer RTJ, Refojo JM, Gallagher PT. 2010. Propagation of an Earth-directed coronal mass ejection in three dimensions. Nat. Commun. 1(6): 74.

Chané E, Jacobs C, Holst BVD, Poedts S, Kimpe D. 2005. On the effect of the initial magnetic polarity and of the background wind on the evolution of CME shocks. A\&A 432(1): 331-339.

Chané E, Holst BVD, Jacobs C, Poedts S, Kimpe D. 2006. Inverse and normal coronal mass ejections: Evolution up to 1 AU. $A \& A$ 447(2): 727-733.

Crooker NU, Larson DE, Kahler SW, Lamassa SM, Spence HE. 2003. Suprathermal electron isotropy in high-beta solar wind and its role in heat flux dropouts. Geophys Res Lett 30(12): 5.

Davis CJ, Davies JA, Lockwood M, Rouillard AP, Eyles CJ, Harrison RA. 2009. Stereoscopic imaging of an Earth-impacting solar coronal mass ejection: A major milestone for the STEREO mission. Geophys Res Lett 36(8): 134-150.

Deforest C, Howard T, Tappin J. 2011. Observations of detailed structure in the solar wind at $1 \mathrm{AU}$ with STEREO/HI-2. Astrophys $J$ 738(1): 103.

Deforest CE, Howard TA, Mccomas DJ. 2012. Disconnecting open solar magnetic flux. Astrophys $J$ 745(1): 36 .

Deforest CE, Howard TA, Mccomas DJ. 2013. Tracking coronal features from the low corona to Earth: A quantitative analysis of the 2008 December 12 coronal mass ejection. Astrophys $J$ 769(769): 43. 
Feldman WC, Asbridge JR, Bame SJ, Gosling JT, Lemons DS. 1978. Characteristic electron variations across simple high-speed solar wind streams. J Geophys Res Space Phys 83(A11): 5285-5295.

Feng X, Yang L, Xiang C, Wu ST, Zhou Y, Zhong DK. 2010. Threedimensional solar WIND modeling from the Sun to Earth by a SIP-CESE MHD model with a six-component grid. Astrophys $J$ 723(1): 300.

Feng X, Zhang M, Zhou Y. 2014. A new three-dimensional solar wind model in spherical coordinates with a six-component grid. Astrophys J Suppl 214(1): 6.

Gibson SE, Fan Y. 2008. Partially ejected flux ropes: Implications for interplanetary coronal mass ejections. J Geophys Res Space Phys 113(A9): A09,103.

Gopalswamy N, Dal LA, Yashiro S, Akiyama S. 2009. The expansion and radial speeds of coronal mass ejections. Cent Eur Astrophys Bull 33(16): 115-124.

Gopalswamy N, Mäkelä P, Yashiro S, Davila JM. 2012. The relationship between the expansion speed and radial speed of CMEs confirmed using quadrature observations of the 2011 February 15 CME. Sun Geosph 7(1): 7-11.

Gosling JT, Koning CAD, Skoug RM, Steinberg JT, Mccomas DJ. 2004. Dispersionless modulations in low-energy solar electron bursts and discontinuous changes in the solar wind electron strahl. J Geophys Res Space Phys 109(A5): 1-12.

Han SM, Wu ST, Dryer M. 1988. A three-dimensional, timedependent numerical modeling of super-sonic, super-alfvénic MHD flow. Comput Fluids 16(1): 81-103.

Hanawa T, Mikami H, Matsumoto T. 2008. Improving shock irregularities based on the characteristics of the MHD equations. $J$ Comput Phys 227(16): 7952-7976.

Hess P, Jie Z. 2017. A study of the Earth-affecting CMEs of solar cycle 24. Sol Phys 292(6): 80.

Howard TA, Deforest CE. 2012. Inner heliospheric flux rope evolution via imaging of coronal mass ejections. Astrophys $J$ 746(1): 812-819.

Iv WBM, Gombosi TI, Roussev I, Ridley A, Zeeuw DLD, Sokolov IV, Powell KG, Tóth G. 2004a. Modeling a space weather event from the Sun to the Earth: CME generation and interplanetary propagation. J Geophys Res Space Phys 109(A2): A02,107.

Iv WBM, Gombosi TI, Roussev I, Zeeuw DLD, Sokolov IV, Powell KG, Tóth G, Opher M. 2004b. Three-dimensional MHD simulation of a flux rope driven CME. J Geophys Res 109(A1): A01,102.

Iv WBM, Ridley AJ, Gombosi TI, Dezeeuw DL. 2006. Modeling the Sun-to-Earth propagation of a very fast CME. Adv Space Res 38(2): 253-262.

Jacobs C, Poedts S. 2011. Models for coronal mass ejections. $J$ Atmos Sol-Terr Phys 73(10): 1148-1155.

Jacobs C, van der Holst B, Poedts S. 2007. Comparison between $2.5 \mathrm{D}$ and $3 \mathrm{D}$ simulations of coronal mass ejections. $A \& A$ 470(1): 359-365.

Kajdić P, Alexandrova O, Maksimovic M, Lacombe C, Fazakerley AN. 2016. Suprathermal electron strahl widths in the presence of narrow-band whistler waves in the solar wind. Astrophys $J$ 833(2): 172.

Kataoka R, Ebisuzaki T, Kusano K, Shiota D, Inoue S, Yamamoto TT, Tokumaru M. 2009. Three-dimensional MHD modeling of the solar wind structures associated with 13 December 2006 coronal mass ejection. J Geophys Res Space Phys 114(A10): A10,102.

Lago A, Schwenn R, Gonzalez W. 2003. Relation between the radial speed and theexpansion speed of coronal mass ejections. Adv Space Res 32(12): 2637-2640.
Lugaz N. 2010. Accuracy and limitations of fitting and stereoscopic methods to determine the direction of coronal mass ejections from heliospheric imagers observations. Sol Phys 267(2): 411-429.

Lugaz N, Manchester WB, Roussev II, Tóth G, Gombosi TI. 2007. Numerical investigation of the homologous coronal mass ejection events from active region 9236. Astrophys $J$ 659(1): 788-800.

Lugaz N, Downs C, Shibata K, Roussev II, Asai A, Gombosi T. 2011. Numerical investigation of a coronal mass ejection from an anemone active region: Reconnection and deflection of the 2005 August 22 eruption. Astrophys $J$ 738(2): 1161-1166.

Mäkelä P, Gopalswamy N, Yashiro S. 2016. The radial speedexpansion speed relation for Earth-directed CMEs. SpaceWeather 14: $368-378$.

Mccomas DJ, Gosling JT, Phillips JL, Bame SJ, Luhmann JG, Smith EJ. 1989. Electron heat flux dropouts in the solar wind-evidence for interplanetary magnetic field reconnection. J Geophys Res Space Phys 94(A6): 6907-6916.

Odstrćil D, Pizzo VJ. 1999a. Three dimensional propagation of coronal mass ejections (CMEs) in a structured solar wind flow: 1. CME launched within the streamer belt. J Geophys Res Space Phys 104: 483-492.

Odstrćil D, Pizzo VJ. 1999b. Three dimensional propagation of coronal mass ejections (CMEs) in a structured solar wind flow: 2. CME launched adjacent to the streamer belt. J Geophys Res Space Phys 104: 493-504.

Odstrćil D, Pizzo VJ, Arge CN. 2005. Propagation of the 12 May 1997 interplanetary coronal mass ejection in evolving solar wind structures. J Geophys Res Space Phys 110(A2): A02,106.

Owens MJ, Crooker NU, Schwadron NA. 2008. Suprathermal electron evolution in a Parker spiral magnetic field. J Geophys Res Space Phys 113(A11): 1-8.

Parker EN. 1963. Interplanetary dynamical processes, Interscience Publishers, New York, NY.

Phillips JL, Gosling JT, Mccomas DJ, Bame SJ, Gary SP, Smith EJ. 2012. Anisotropic thermal electron distributions in the solar wind. J Geophys Res Space Phys 94(A6): 6563-6579.

Riley P, Crooker NU. 2004. Kinematic treatment of coronal mass ejection evolution in the solar wind. Astrophys J 600: 1035-1042.

Shen F, Shen C, Zhang J, Hess P, Wang Y, Feng X, Cheng H, Yang Y. 2014. Evolution of the 2012 July 12 CME from the Sun to the Earth: Data-constrained three-dimensional MHD simulations. $J$ Geophys Res Space Phys 119(9): 7128-7141.

Shiota D, Kataoka R. 2016. Magnetohydrodynamic simulation of interplanetary propagation of multiple coronal mass ejections with internal magnetic flux rope (SUSANOO-CME). Space Weather Int $J$ Res Appl 14(2): 56-75.

Vandas M, Odstrćil D, Watari S. 2002. Three-dimensional MHD simulation of a loop-like magnetic cloud in the solar wind. $J$ Geophys Res Space Phys 107(A9): 1236.

Wada W, Liou MS. 2012. An accurate and robust flux splitting scheme for shock and contact discontinuities. Siam J Sci Comput 18(3): 633-657.

Wang R, Lu Q, Du A, Wang S. 2010a. In situ observations of a secondary magnetic island in an ion diffusion region and associated energetic electrons. Phys Rev Lett 104(17): 175,003.

Wang R, Lu Q, Li X, Huang C, Wang S. 2010b. Observations of energetic electrons up to $200 \mathrm{keV}$ associated with a secondary island near the center of an ion diffusion region: A cluster case study. J Geophys Res Atmos 115(A11): 11,201.

Wu CC, Fry CD, Dryer M, Wu ST, Thompson B, Kan L, Feng XS. 2007a. Three-dimensional global simulation of multiple ICMEs interaction and propagation from the Sun to the heliosphere 
following the 25C28 October 2003 solar events. Adv Space Res 40(12): 1827-1834.

Wu CC, Fry CD, Wu ST, Dryer M, Kan L. 2007b. Threedimensional global simulation of interplanetary coronal mass ejection propagation from the Sun to the heliosphere: Solar event of 12 May 1997. J Geophys Res Space Phys 112(A9): A09,104.

Ying L, Davies JA, Luhmann JG, Vourlidas A, Bale SD, Lin RP. 2010. Geometric triangulation of imaging observations to track coronal mass ejections continuously out to 1 AU. Astrophys $J$ 710(1): L82-L87.

Zhou YF, Feng XS. 2013. MHD numerical study of the latitudinal deflection of coronal mass ejection. J Geophys Res Space Phys 118(10): 6007-6018.

Zhou YF, Feng XS, Wu ST, Du D, Shen F, Xiang CQ. 2012. Using a 3-D spherical plasmoid to interpret the Sun-to-Earth propagation of the 4 November 1997 coronal mass ejection event. J Geophys Res 117(A01): 102.

Ziegler U. 2004. A central-constrained transport scheme for ideal magnetohydrodynamics. J Comput Phys 196: 393-416.

Ziegler U. 2011. A semi-discrete central scheme for magnetohydrodynamics on orthogonal-curvilinear grids. J Comput Phys 230: 1035-1063.

Zuccarello FP, Bemporad A, Jacobs C, Mierla M, Poedts S, Zuccarello F. 2012a. The role of streamers in the deflection of coronal mass ejections: Comparison between stereo three-dimensional reconstructions and numerical simulations. Astrophys $J$ 744(66): 14.

Zuccarello FP, Meliani Z, Poedts S. 2012b. Numerical modeling of the initiation of coronal mass ejections in active region NOAA 9415. Astrophys J 758(2): 117.

Cite this article as: Zhang M, Feng X \& Yang L 2019. Three-dimensional MHD simulation of the 2008 December 12 coronal mass ejection: from the Sun to Interplanetary space. J. Space Weather Space Clim. 9, A33. 